\title{
A BEM SENSITIVITY FORMULATION FOR 3D ACTIVE NOISE CONTROL
}

\author{
A. BRANCATI*, M. H. ALIABADI* ${ }^{*}$ V. MALLARDO*,1
}

\begin{abstract}
In this paper a novel Boundary Element (BE) formulation for sensitivity analysis of local active noise control in a three-dimensional field is presented. The formulation is based on the Helmholtz differential equation and it is valid for internal as well as for scattering wave propagation problems. The primary noise is attenuated within an enclosure, called control volume, by adding a control source modelled as an object with a vibrating surface. Both the optimum position of the control volume and the optimum location/orientation of the secondary source are determined by minimisation of a suitable cost function. The sensitivities are determined by implicit differentiation. A hierarchical adaptive cross approximation approach in conjunction with the GMRES solver is implemented to accelerate the convergence. Four numerical examples are presented to demonstrate accuracy and efficiency of the proposed formulations.
\end{abstract}

\section{INTRODUCTION}

In the last few decades Active Noise Control (ANC) has been widely explored. Early research into global noise reduction in a free space involved the use of secondary loudspeakers placed close to the primary sources (Conover [1], Nelson et al. [2,3], Ross [4], Hesselman [5], Berge et al. [6]). In an enclosed space, a global noise reduction is achieved only for frequencies close to resonance and the

Key words and phrases. Boundary Element Method, Sensitivity analysis, Adaptive Cross Approximation/Hierarchical matrix format, Optimum secondary source location/orientation, Extended noise attenuation volume.

*Department of Aeronautics, Imperial College London, South Kensington, SW7 2AZ, UK, a.brancati06@imperial.ac.uk

${ }^{1}$ on leave from the Department of Architecture, University of Ferrara, Italy

emails. a.brancati06@imperial.ac.uk,m.h.aliabadi@imperial.ac.uk,mlv@unife.it. 
secondary source can be located everywhere in enclosures except at nodal points (Nelson et al. [2]). Elliott et al. [7-9] demonstrated the feasibility of a system that attenuates the noise in a local sense.

Recently, techniques aimed at minimising the noise level by the optimisation of the actuator locations have been explored. Padula et al. [10] reviewed optimization techniques for the Active Structural Acoustic Control (ASAC) for both actuator (shakers or piezoelectric) and sensor locations, and summarized experimental and numerical results demonstrating the benefit of using numerical methods. Design sensitivity analysis and optimisation techniques related to structural-acoustic coupling are reviewed in [11,12]. The work of Ruckman and Fuller [13] represents an early application to ASAC. In their investigation they adopted an approach where structural control actuator locations are optimized over a subset selection (i.e., only a discrete number of locations is permitted). In [14], Kincaid et al. evaluated the optimum force inputs and locations for piezo-ceramic actuators bonded to the wall of a vibrating cylinder simulating an aircraft fuselage.

The work of Baek and Elliott [15] represents an early ANC investigation of optimum location of speakers achieved by using genetic algorithms and simulated annealing methods. The book by Koopman and Fahnline [16] presents a comprehensive study on passive and active noise control. Martin and Roure [17] applied a mixed method using a selective search algorithm for the optimum location of the actuators and a genetic algorithm to determine the optimum location of the error sensors. Kincaid et al. [18] attempted to obtain the optimum locations of a group of sensors for an ANC application using a Reactive Tabu Search (RTS). Their numerical model was validated against laboratory results. Seyedin and Abedi [19] simulated the sound propagation in a room with reflecting surfaces and obtained the optimum locations of an anti-noise source, which is a monopole constrained at one of the four walls.

Christensen and Olhoff [20] optimised the directivity of the sound emission from the diaphragm of an electro dynamic loudspeaker using a coupled Finite Element Method (FEM) and Boundary Element Method (BEM). 
Methods such as genetic algorithms and simulated annealing generally lack a mathematical proof of their convergence and tend to require large number of computations of error functions. In this paper an alternative approach based on the exact implicit evaluation of the sensitivity parameters for the optimisation problem is proposed using a boundary element method. The proposed BEM is coupled with the Adaptive Cross Approximation (ACA), the Hierarchical matrix ( $\mathcal{H}$-matrix) format and the GMRES [21] to allow fast solutions for large scale problems. Previous work on BEM sensitivities can be found in [22-28].

An early investigation of the BEM sensitivity analysis in the ANC context can be found in [29]. It minimises the total radiated power emanating from a pulsating sphere and from vibrating surfaces within a box using the secondary source vibration surface velocity. A study by Yang and Tseng [30] was mainly focused on the optimal position of loudspeakers in 2D and 3D cases. The indirect BEM was used to simulate the sound propagation while the sequential quadratic programming (SQP) was selected as optimizer. Bai and Chang [31] reduced a noise radiated in enclosures with specific acoustic impedances. The total time average acoustic potential energy was selected as the cost function to be minimised and used to optimize the positions and the amplitudes of the secondary sources. All the above studies used the so-called "zero ${ }^{\text {th }}$ order optimisation".

The first order optimisation methods need the first derivatives of the cost function. In the BEM context the problem can be reduced to the solutions of two systems of equations, one for the direct problem and another for the sensitivities. A key issue is the computational effort related to the optimisation procedure. Prasad and Kane [32] demonstrated the superiority of employing preconditioned iterative equation solvers for sensitivity analysis. Fritze et al. [33] proposed a coupled FE (structure) and BE (external fluid) approach for passive noise control of large scale models. Nemitz and Bonnet [34] utilised a sensitivity based boundary element formulation accelerated by the fast multipole method to solve the problem of inverse scattering of scalar waves.

In this paper a BEM formulation for the optimisation of a local ANC is presented. The proposed noise attenuation strategy consists of minimising a cost 
function proportional to the total time averaged acoustic potential energy [35] within a predefined volume, called Control Volume (CV), by a secondary source. An implicit BEM sensitivity formulation is developed for finding the optimum locations of both the CV and the control source as well as the optimum orientation of the control source. Furthermore, the computational cost of the proposed analysis is reduced by adopting the ACA technique coupled with the $\mathcal{H}$-matrix format and the GMRES.

The BEM is applied to the three dimensional (3D) Helmholtz equation [36,37] for monotone frequencies both to determine the solution of the direct problem and to evaluate the sensitivities by an implicit differentiation approach. The direct solution and the sensitivities are used to solve the optimisation problem. A superparametric formulation with linear and constant elements has been utilised. The Adaptive Cross Approximation (ACA) is used to generate both the system matrix and the right hand side vector, the $\mathcal{H}$-matrix format is used for the storage requirements and the GMRES is used to solve the linear systems of equations. The same strategy is also used to evaluate the potential at selected internal points, and the design sensitivities on the boundary and in the internal domain. Several examples are presented to demonstrate the accuracy of the proposed procedure. They include:

i) optimum CV location in an infinite domain;

ii) optimum secondary source location inside a room;

iii) optimum secondary source orientation inside a box;

iv) optimum secondary source locations and orientations at different frequencies in an aircraft cabin.

\section{LOCAL ACTIVE NOISE CONTROL APPROACH}

In this paper the ANC has been developed by attenuating an unwanted noise in a confined region. The approach consists of reducing the noise inside an area of interest (i.e., the $\mathrm{CV}$ ) denoted as $D$.

In general acoustic simulations, the field is formulated using the linear wave equation that in case of time-harmonic wave motion the solution can be evaluated 
as the product of two functions, the first depends only upon the space (i.e., the velocity potential $U(\mathbf{x})$, here referred simply to potential) and the second only upon the time (i.e., $\left.e^{i \omega t}\right)$. Due to this hypothesis the acoustic field can be described by the Helmholtz equation as

$$
\nabla^{2} U(\mathbf{x})+k^{2} U(\mathbf{x})=\frac{1}{c^{2}} b
$$

where $k=\omega / c$, with $\omega$ angular frequency and $c$ sound velocity, is the wavenumber; $b$ refers to the presence of sources within the domain $\Omega$ with strength $b\left(\mathbf{X}^{\mathbf{s}}\right) / c^{2}$.

The noise level within $D$ is reduced by minimising the integral of the square modulus of the total potential $U\left(\mathbf{X}_{D}\right)$ at the points in the volume $D$

$$
f_{c}\left(\mathbf{X}_{D}\right)=\int_{D}\left|U\left(\mathbf{X}_{D}^{\prime}\right)\right|^{2} d D
$$

with $\mathbf{X}_{D}^{\prime} \in D$. It can be demonstrated (see $[31,38]$ ) that such a function is proportional to the total time averaged acoustic potential energy.

The above cost function (2) is minimised, for a given disturbing primary noise, by the secondary source field, which is generated by a $3 \mathrm{D}$ rigid object (i.e., $q=0$ ) with a vibrating portion (i.e., $q \neq 0$ ) (see figure 3 ). The optimum velocity of this portion $\overline{\mathbf{q}}_{s}\left(\mathbf{x}_{v}\right)$ is evaluated by an amplifying factor $\alpha$, which relates the optimum secondary field solution to a solution obtained by any velocity of secondary source vibrating surface $\mathbf{q}_{s}\left(\mathbf{x}_{v}\right)$, i.e.,

$$
\overline{\mathbf{q}}_{s}\left(\mathbf{x}_{v}\right)=\alpha \mathbf{q}_{s}\left(\mathbf{x}_{v}\right)
$$

where $\mathbf{x}_{v}$ refers to the points of the secondary vibrating surface.

Since the acoustic field is linear, the potential $U$ at any point inside the domain can be viewed as the superposition of the primary and secondary fields (evaluated separately). Then, the problem is solved by minimising the cost function (2) with respect to the parameter $\alpha$ (i.e., setting the cost function derivative with the 
respect to both the real and imaginary parts equal to zero) and the solution can be written as follows

$$
\alpha=\left(-\frac{\bar{c}+\bar{d}}{\bar{a}+\bar{b}}, \frac{\bar{f}-\bar{g}}{\bar{a}+\bar{b}}\right)
$$

The constant values in the above expression are shown in table 1 , in which the subscripts ${ }_{p}$ and ${ }_{s}$ refer to the primary and secondary quantities, respectively, and the subscripts $R$ and ${ }_{I}$ refer to the real and imaginary parts, respectively.

\begin{tabular}{cccccc}
\hline $\bar{a}$ & $b$ & $\bar{c}$ & $d$ & $f$ & $\bar{g}$ \\
\hline $\int_{D} U_{s R}^{2} d D$ & $\int_{D} U_{s I}^{2} d D$ & $\int_{D} U_{p R} U_{s R} d D$ & $\int_{D} U_{p I} U_{s I} d D$ & $\int_{D} U_{p R} U_{s I} d D$ & $\int_{D} U_{p I} U_{s R} d D$ \\
\hline
\end{tabular}

TABLE 1. Values of the terms in Eq. (4).

\section{OPTIMISATION STRATEGY}

The present essay has two goals: $i$ ) to optimize both the location and the orientation of the secondary source by keeping the CV fixed (see figure 1), and $i i$ ) to optimize the $\mathrm{CV}$ location by fixing the control source position (see figure 2). 


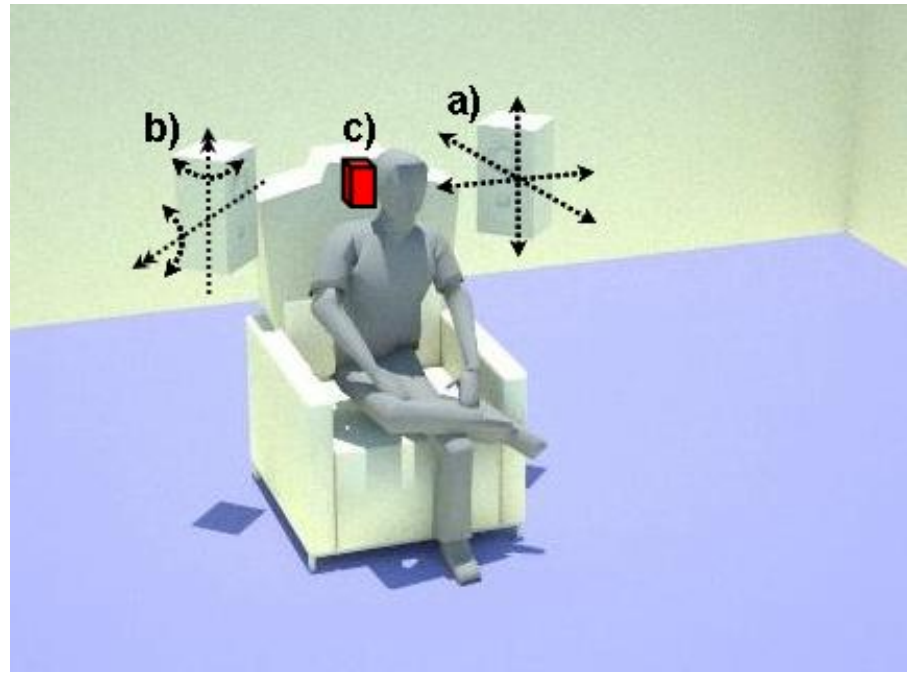

FigURE 1. Optimisation process applied to the control source: a) optimum location; b) optimum orientation; c) fixed CV.

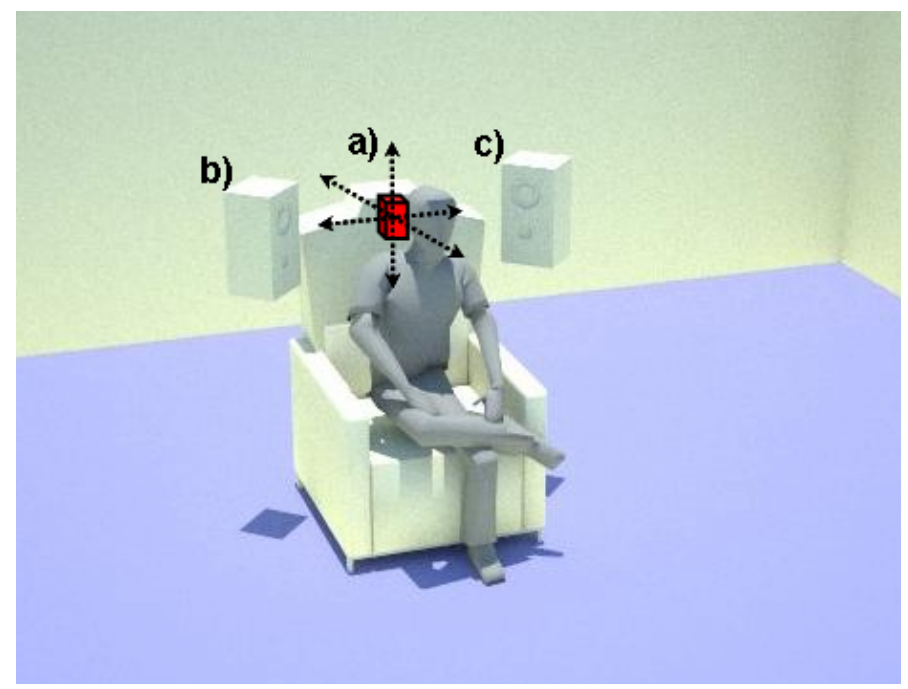

Figure 2. Optimisation process applied to the CV: a) optimum CV location; b-c) fixed control sources. 
The cost function (2) depends thus on the position vectors of the $\mathrm{CV} \mathbf{a} \equiv a_{l}=$ $\left(a_{1}, a_{2}, a_{3}\right)$ and of the secondary source $\mathbf{b} \equiv b_{m}=\left(b_{1}, b_{2}, b_{3}\right)$ (see figure 3$)$.

The CV location is optimized by assuming that its shape and orientation remain fixed from the initial location to the final and that the secondary source is unmoved. Hence, the design variable is $\mathbf{a}$ with $\mathbf{b}$ fixed. On the contrary during the optimum control source location/orientation optimisation procedure the design variable is $\mathbf{b}$ with $\mathbf{a}$ fixed.

The optimisation problems can be solved iteratively and the final solution is achieved if the difference between the cost functions $\left(f_{c}(\mathbf{a})\right.$ or $\left.f_{c}(\mathbf{b})\right)$ at two consecutive design variables is below a prescribed positive tolerance $\varepsilon$ [39]

$$
\frac{\left|f\left(\mathbf{a}^{k+1}\right)-f\left(\mathbf{a}^{k}\right)\right|}{\left|f\left(\mathbf{a}^{k}\right)\right|}<\varepsilon
$$

where $k$ and $k+1$ represent the iteration numbers of two consecutive iterations.

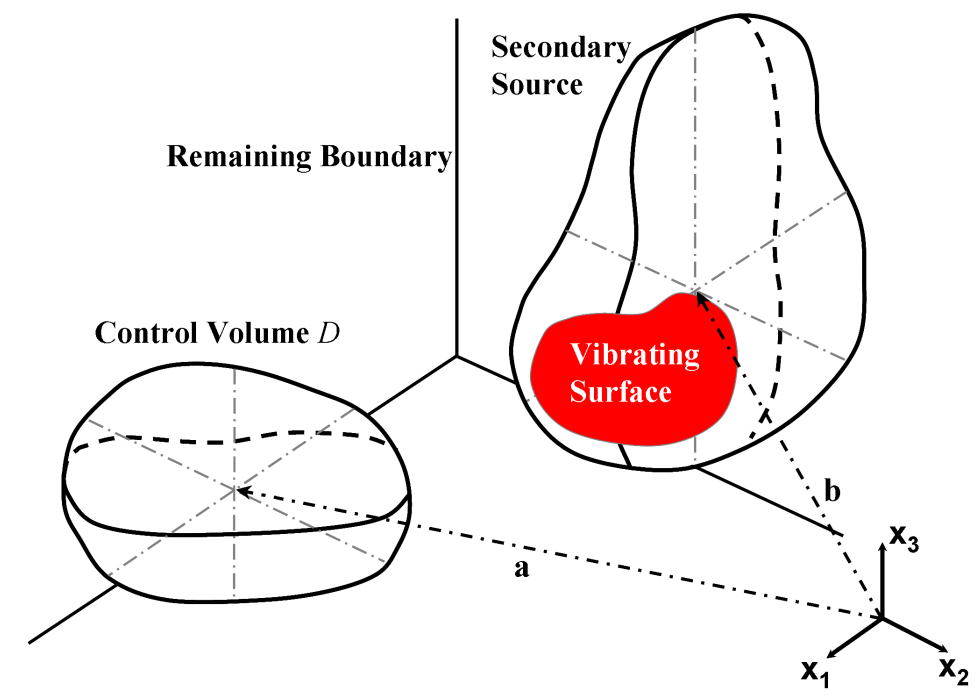

FiguRE 3. Geometry of the problem: secondary source boundary, remaining boundary, $\mathrm{CV}$, secondary source vibrating surface, position vectors of the $\mathrm{CV} \mathbf{a} \equiv a_{l}=\left(a_{1}, a_{2}, a_{3}\right)$ and of the secondary source $\mathbf{b} \equiv b_{m}=\left(b_{1}, b_{2}, b_{3}\right)$. 
The design variable vector $\mathbf{a}$ (or $\mathbf{b}$ ) at the $k+1$ iteration can be evaluated by the value of the vector at the $k^{\prime}$ th iteration as follows

$$
\mathbf{a}^{k+1}=\mathbf{a}^{k}+\Delta \mathbf{a}^{k}
$$

where $\Delta \mathbf{a}^{k}$ is a variation of the current point and it can be represented as

$$
\Delta \mathbf{a}^{k}=\alpha_{k} \mathbf{d}^{k}
$$

where $\mathbf{d}^{k}$ is the best search direction to seek the optimum solution and $\alpha_{k}$ is the step size, a positive scalar quantity, that indicates the step in that direction. In practice, the optimisation process is converted into two sub-problems, i.e., one related to evaluation of the best direction to modify the design variables and the second associated to the determination of the step length. These two quantities are iteratively updated to evaluate the optimum design variable until the condition (5) is fulfilled. In this study the tolerance is set as $\varepsilon=10^{-8}$.

The optimisation procedure adopted here is based upon the Broyden-FletcherGoldfarb-Shanno (BFGS) variant of the Davidon-Fletcher-Powell (DFP) method $[39,40]$ that is a first order method.

Hence, at each iteration the derivative of the cost function (2) with respect to each single component $m$ of a design variable (either $\mathbf{a}$ or $\mathbf{b}$ ) is required and it is evaluated using equation (2) as follows

$$
\text { (8) } \begin{aligned}
f_{c, m}(\mathbf{a}, \mathbf{b}) & =\frac{\partial f_{c}(\mathbf{a}, \mathbf{b})}{\partial m}= \\
= & \int_{D} \frac{\partial}{\partial a_{m}}\left[\left(U_{p R}+\alpha_{R} U_{s R}-\alpha_{I} U_{s I}\right)^{2}+\left(U_{p I}+\alpha_{I} U_{s R}+\alpha_{R} U_{s I}\right)^{2}\right] d D
\end{aligned}
$$

where the Leibniz Integral Rule has been used. The two additional terms, that are generated if the limits of integration are functions of the design variable, have not been included in equation (8) because the CV geometry is not modified by the differentiation process. 
Manipulating equation (8) yields the following explicit expression

$$
\begin{gathered}
f_{c, m}(\mathbf{a}, \mathbf{b})=2 \int_{D}\left[( U _ { p R } + \alpha _ { R } U _ { s R } - \alpha _ { I } U _ { s I } ) \left(U_{p R, m}+\alpha_{R, m} U_{s R}+\alpha_{R} U_{s R, m}-\alpha_{I, m} U_{s I}-\right.\right. \\
\left.\left.\alpha_{I} U_{s I, m}\right)+\left(U_{p I}+\alpha_{I} U_{s R}+\alpha_{R} U_{s I}\right)\left(U_{p I, m}+\alpha_{I, m} U_{s R}+\alpha_{I} U_{s R, m}-\alpha_{R, m} U_{s I}-\alpha_{R} U_{s I, m}\right)\right] d D
\end{gathered}
$$

where $\alpha_{R, m}$ and $\alpha_{I, m}$ are evaluated by considering the value of $\alpha$ that provides the optimum secondary response with $\mathbf{a}$ and $\mathbf{b}$ given.

Therefore, the optimisation process can be updated if the sensitivities are evaluated at every iteration.

\section{IMPLICIT DIFFERENTIATION OF THE BOUNDARY INTEGRAL EQUATION}

The sensitivities involved in equation (9) can be determined by the classic boundary integral equation collocated at the boundary point $\mathbf{x}^{\prime}$, i.e.,

$$
c\left(\mathbf{x}^{\prime}\right) u\left(\mathbf{x}^{\prime}\right)+\int_{\Gamma} u(\mathbf{x}) q^{*}\left(\mathbf{x}^{\prime}, \mathbf{x}\right) d \Gamma=\int_{\Gamma} q(\mathbf{x}) u^{*}\left(\mathbf{x}^{\prime}, \mathbf{x}\right) d \Gamma+\sum_{t=1}^{N P} P_{t}\left(\mathbf{x}^{\prime}, \mathbf{X}_{t}^{s}\right)
$$

where $c\left(\mathbf{x}^{\prime}\right)$ is the free terns; $u(\mathbf{x})$ and $q(\mathbf{x})$ are the potential and the flux at the boundary and $P_{t}\left(\mathbf{x}_{j}^{\prime}, \mathbf{X}_{t}^{s}\right)$ refers to the presence of the $t^{\prime} t h$ extra source located at $\mathbf{X}_{t}^{s}$. Finally, $u^{*}\left(\mathbf{x}^{\prime}, \mathbf{x}\right)$ and $q^{*}\left(\mathbf{x}^{\prime}, \mathbf{x}\right)$ are the potential and the flux fundamental solutions, respectively.

The differentiation of equation (10) with respect to the $m^{\prime} t h$ design variable can be written as follows

$$
\begin{aligned}
& c\left(\mathbf{x}^{\prime}\right) u_{, m}\left(\mathbf{x}^{\prime}\right)+\int_{\Gamma} u_{, m}(\mathbf{x}) q^{*}\left(\mathbf{x}^{\prime}, \mathbf{x}\right) d \Gamma+\int_{\Gamma} u(\mathbf{x}) q_{, m}^{*}\left(\mathbf{x}^{\prime}, \mathbf{x}\right) d \Gamma= \\
& \int_{\Gamma} q_{, m}(\mathbf{x}) u^{*}\left(\mathbf{x}^{\prime}, \mathbf{x}\right) d \Gamma+\int_{\Gamma} q(\mathbf{x}) u_{, m}^{*}\left(\mathbf{x}^{\prime}, \mathbf{x}\right) d \Gamma+\sum_{t=1}^{N P} P_{t, m}\left(\mathbf{x}^{\prime}, \mathbf{X}_{t}^{s}\right)
\end{aligned}
$$


where $c_{, m}\left(\mathbf{x}_{j}^{\prime}\right)$ is zero as the design variables do not affect smoothness of the boundary elements.

The discretised forms of the equations (10) and (11) can be written as follows

$$
\begin{aligned}
c\left(\mathbf{x}_{j}^{\prime}\right) u\left(\mathbf{x}_{j}^{\prime}\right)+\sum_{i=1}^{N} u_{i} \int_{-1}^{+1} \int_{-1}^{+1} q^{*}\left(\mathbf{x}_{j}^{\prime}, \mathbf{x}\left(\eta_{k}\right)\right) J_{i} d \eta_{1} d \eta_{2}= & \\
& \sum_{i=1}^{N} q_{i} \int_{-1}^{+1} \int_{-1}^{+1} u^{*}\left(\mathbf{x}_{j}^{\prime}, \mathbf{x}\left(\eta_{k}\right)\right) J_{i} d \eta_{1} d \eta_{2}+\sum_{t=1}^{N P} P_{t}\left(\mathbf{x}_{j}^{\prime}, \mathbf{X}_{t}^{s}\right)
\end{aligned}
$$

and

$$
c\left(\mathbf{x}_{j}^{\prime}\right) u_{, m}\left(\mathbf{x}_{j}^{\prime}\right)+\sum_{i=1}^{N} u_{i, m} \int_{-1}^{+1} \int_{-1}^{+1} q^{*}\left(\mathbf{x}_{j}^{\prime}, \mathbf{x}\left(\eta_{k}\right)\right) J_{i} d \eta_{1} d \eta_{2}+
$$

$$
\begin{array}{r}
\sum_{i=1}^{N} u_{i} \int_{-1}^{+1} \int_{-1}^{+1} q_{, m}^{*}\left(\mathbf{x}_{j}^{\prime}, \mathbf{x}\left(\eta_{k}\right)\right) J_{i} d \eta_{1} d \eta_{2}=\sum_{i=1}^{N} q_{i, m} \int_{-1}^{+1} \int_{-1}^{+1} u^{*}\left(\mathbf{x}_{j}^{\prime}, \mathbf{x}\left(\eta_{k}\right)\right) J_{i} d \eta_{1} d \eta_{2}+ \\
\sum_{i=1}^{N} q_{i} \int_{-1}^{+1} \int_{-1}^{+1} u_{, m}^{*}\left(\mathbf{x}_{j}^{\prime}, \mathbf{x}\left(\eta_{k}\right)\right) J_{i} d \eta_{1} d \eta_{2}+\sum_{t=1}^{N P} P_{t, m}\left(\mathbf{x}_{j}^{\prime}, \mathbf{X}_{t}^{s}\right)
\end{array}
$$

where $\mathbf{x}_{j}^{\prime}$ denotes the collocation point; $k=1,2 ; j=1, \ldots N$ with $N$ number of boundary nodes; $u_{i}$ and $q_{i}$ are the potential and the flux at the element $i$, respectively; $\eta_{1}, \eta_{2}$ are the local coordinates and $J_{i}$ is the Jacobian of the $i^{\prime} t h$ integration element.

It should be noted that in equation (13) the derivative of the Jacobian with respect to the design variable, $J_{, m}$, is zero as the optimisation process do not modify the boundary elements.

The value of the potential at any internal point $\mathbf{X}^{\prime}$ can be obtained in terms of potential $u$ and flux $q$ values at the boundary elements, i.e., 


$$
U\left(\mathbf{X}^{\prime}\right)=-\int_{\Gamma} u(\mathbf{x}) q^{*}\left(\mathbf{X}^{\prime}, \mathbf{x}\right) d \Gamma+\int_{\Gamma} q(\mathbf{x}) u^{*}\left(\mathbf{X}^{\prime}, \mathbf{x}\right) d \Gamma+\sum_{t=1}^{N P} P_{t}\left(\mathbf{X}^{\prime}, \mathbf{X}_{t}^{s}\right)
$$

whose derivative can be written as follows

$$
\begin{aligned}
U\left(\mathbf{X}^{\prime}\right)_{, m}= & -\int_{\Gamma} u_{, m}(\mathbf{x}) q^{*}\left(\mathbf{X}^{\prime}, \mathbf{x}\right) d \Gamma-\int_{\Gamma} u(\mathbf{x}) q_{, m}^{*}\left(\mathbf{X}^{\prime}, \mathbf{x}\right) d \Gamma+ \\
& \int_{\Gamma} q_{, m}(\mathbf{x}) u^{*}\left(\mathbf{X}^{\prime}, \mathbf{x}\right) d \Gamma+\int_{\Gamma} q(\mathbf{x}) u_{, m}^{*}\left(\mathbf{X}^{\prime}, \mathbf{x}\right) d \Gamma+\sum_{t=1}^{N P} P_{t, m}\left(\mathbf{X}_{j}^{\prime}, \mathbf{X}_{t}^{s}\right)
\end{aligned}
$$

The discretised forms of the equations (14) and (15) can be written as follows

$$
\begin{aligned}
U\left(\mathbf{X}_{j}^{\prime}\right)=-\sum_{i=1}^{N} u_{i} & \int_{-1}^{+1} \int_{-1}^{+1} q^{*}\left(\mathbf{X}_{j}^{\prime}, \mathbf{x}\left(\eta_{k}\right)\right) J_{i} d \eta_{1} d \eta_{2}+ \\
& \sum_{i=1}^{N} q_{i} \int_{-1}^{+1} \int_{-1}^{+1} u^{*}\left(\mathbf{X}_{j}^{\prime}, \mathbf{x}\left(\eta_{k}\right)\right) J_{i} d \eta_{1} d \eta_{2}+\sum_{t=1}^{N P} P_{t}\left(\mathbf{X}_{j}^{\prime}, \mathbf{X}_{t}^{s}\right)
\end{aligned}
$$

and

$$
\begin{aligned}
& \text { (17) } U\left(\mathbf{X}_{j}^{\prime}\right)_{, m}=-\sum_{i=1}^{N} u_{i, m} \int_{-1}^{+1} \int_{-1}^{+1} q^{*}\left(\mathbf{X}_{j}^{\prime}, \mathbf{x}\left(\eta_{k}\right)\right) J_{i} d \eta_{1} d \eta_{2}- \\
& \sum_{i=1}^{N} u_{i} \int_{-1}^{+1} \int_{-1}^{+1} q_{, m}^{*}\left(\mathbf{X}_{j}^{\prime}, \mathbf{x}\left(\eta_{k}\right)\right) J_{i} d \eta_{1} d \eta_{2}+\sum_{i=1}^{N} q_{i, m} \int_{-1}^{+1} \int_{-1}^{+1} u^{*}\left(\mathbf{X}_{j}^{\prime}, \mathbf{x}\left(\eta_{k}\right)\right) J_{i} d \eta_{1} d \eta_{2}+ \\
& \sum_{i=1}^{N} q_{i} \int_{-1}^{+1} \int_{-1}^{+1} u_{, m}^{*}\left(\mathbf{X}_{j}^{\prime}, \mathbf{x}\left(\eta_{k}\right)\right) J_{i} d \eta_{1} d \eta_{2}+\sum_{t=1}^{N P} P_{t, m}\left(\mathbf{X}_{j}^{\prime}, \mathbf{X}_{t}^{s}\right)
\end{aligned}
$$

Equation (13) can be collocated at each boundary point in order to build a final system of equations in terms of the unknown sensitivities. After solving the system by imposing the boundary conditions, the sensitivities at any internal point can be determined by equation (17). It must be pointed out that the above 
relations can be evaluated if both the derivatives of the fundamental solutions and the derivatives of $r$ and of its components with respect to the design variables are obtained. Such terms are outlined in the next section.

\section{SENSITIVITY ANALYSIS}

The potential and flux fundamental solutions are given, respectively, by [36,37]

$$
\begin{gathered}
u^{*}\left(\mathbf{X}^{\prime}, \mathbf{x}\right)=\frac{1}{4 \pi r} \mathbf{e}^{-i k r} \\
q^{*}\left(\mathbf{X}^{\prime}, \mathbf{x}\right)=\frac{\partial u^{*}}{\partial r} \frac{\partial r}{\partial n}=-\frac{1}{4 \pi r^{2}}(1+i k r) \mathbf{e}^{-i k r} r_{, n}
\end{gathered}
$$

where $r$ is the distance between the field point $\mathbf{x}$ and either the internal $\mathbf{X}^{\prime}$ and the boundary $\mathbf{x}^{\prime}$ collocation point, $r=\left|\mathbf{x}-\mathbf{X}^{\prime}\right|\left(\right.$ or $\left.r=\left|\mathbf{x}-\mathbf{x}^{\prime}\right|\right)$, and $i$ is the imaginary unit.

The derivatives of the fundamental solutions can be evaluated as follow

$$
\begin{gathered}
u_{, m}^{*}\left(\mathbf{X}^{\prime}, \mathbf{x}\right)=u_{, r}^{*} r_{, m}=-\frac{1}{4 \pi r^{2}}(1+i k r) \mathbf{e}^{-i k r} r_{, m} \\
q_{, m}^{*}\left(\mathbf{X}^{\prime}, \mathbf{x}\right)=q_{, r}^{*} r_{, m}=\frac{1}{4 \pi r^{3}}\left[\left(2+2 i k r-k^{2} r^{2}\right) r_{, n} r_{, m}-\left(r+i k r^{2}\right) r_{, n m}\right] \mathbf{e}^{-i k r}
\end{gathered}
$$

where the partial derivatives of $r$ and $r_{, n}$ with respect to the design variables $\left(r_{, m}\right.$ and $r_{, n m}$ ) can be calculated using the chain rule. Their expressions depend upon the type of optimisation problem under analysis, i.e., optimum $\mathrm{CV} /$ secondary source location or optimum secondary source orientation.

\subsection{Optimum Secondary Source and CV Locations.}

In case of optimisation of the control source location the value of $r_{, m}$ is evaluated as follows

$$
r_{, m}=r_{, j} r_{j, m}=\frac{r_{j}}{r}\left( \pm \delta_{j m}\right)= \pm \frac{r_{m}}{r}
$$


where $j=1,2,3$ refers to the coordinate system, $\delta_{j m}$ is the Kronecker delta, $r_{j}=x_{j}-x_{j}^{\prime}$ and $r_{m}$ is the component along the direction $m$. The sign of the expression (20) depends upon the element over which the integral is calculated. In particular, if the element belongs to the secondary source, $m$ coincides with the direction of $x_{j}$ and the sign of (20) is positive, whereas if the collocation point is on the secondary source boundary, $m$ coincides with the direction of $x_{j}^{\prime}$ and (20) is negative.

Now, the second derivative of $r$ with respect to $n$ and $m$ is given by the following relation

$$
r_{, n m}= \pm\left(\frac{n_{m}}{r}-\frac{r_{m}\left(r_{j} n_{j}\right)}{r^{3}}\right)
$$

where the sign follows the same rule of equation (20).

In case of optimisation of the $\mathrm{CV}$ location, the problem is solved by equations (20) and (21) that, since $r_{j}=x_{j}-X_{j}^{\prime}$, have always negative signs.

\subsection{Optimum Control Source Orientation.}

The formulation presented in subsection 5.1 is not applicable for evaluating the optimum orientation of the control source. Next, a procedure for dealing with orientation is described.

Figure 4 shows the Euler angles used in the proposed approach. Let $\bar{X}_{j}$ system, with $j=1,2,3$, be the fixed and let the $\bar{x}_{j}$ system rotate with the control source. $N$ is the line of nodes. Both systems have origins in the centre of the secondary source. The first rotation is by an angle $\phi$ around the $\bar{X}_{3}$-axes, the second by an angle $\theta$ around $\bar{x}_{1}$-axes and the last by an angle $\psi$ around $\bar{x}_{3}$-axes.

The transformation matrices $\left(\overline{\mathbf{x}}=\mathbf{R}_{3} \mathbf{R}_{2} \mathbf{R}_{1} \overline{\mathbf{X}}\right)$ are 


$$
\begin{aligned}
& \mathbf{R}_{1}=\left[\begin{array}{ccc}
\cos \phi & \sin \phi & 0 \\
-\sin \phi & \cos \phi & 0 \\
0 & 0 & 1
\end{array}\right] \\
& \mathbf{R}_{2}=\left[\begin{array}{ccc}
1 & 0 & 0 \\
0 & \cos \theta & \sin \theta \\
0 & -\sin \theta & \cos \theta
\end{array}\right] \\
& \mathbf{R}_{3}=\left[\begin{array}{ccc}
\cos \psi & \sin \psi & 0 \\
-\sin \psi & \cos \psi & 0 \\
0 & 0 & 1
\end{array}\right]
\end{aligned}
$$

The partial derivatives with respect to $m(\phi$ or $\theta$ or $\psi)$ of the distance $r$ can be evaluated as follows

$$
r_{, m}=\left[\left(r_{j}^{2}\right)^{1 / 2}\right]_{, m}=\frac{r_{j} r_{j, m}}{r}
$$

where $r_{j, m}$ with $j=1,2,3$ is the derivative of the component $r_{j}$ along the $j$-axes with respect to the design variable $m$. The derivative of $r_{, n}$ with respect to $m$ can be written as follows

$$
r_{, n m}=\frac{\partial}{\partial m} \frac{\partial r}{\partial n}=\frac{n_{j} r_{j, m}+n_{j, m} r_{j}-r_{, n} r_{, m}}{r}
$$

where $n_{j, m}$ indicates the derivative of the outward normal $n_{j}$ along the $j$-axes with respect to the design variable $m$.

In order to evaluate (23) and (24), only the derivative with respect to the design variable of the node coordinates $x_{j}$ in the global coordinate system is required. It should be noted that $r_{j, m}$ coincides with $x_{j, m}$ when the element node belongs to the secondary source boundary, whereas it coincides with $-x_{j, m}$ when the point 


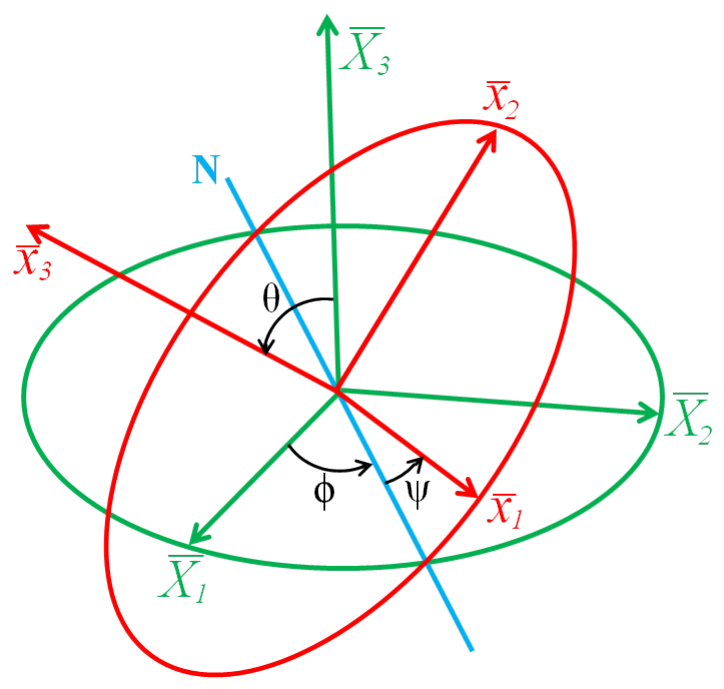

FiguRE 4. Euler angles.

source is on the secondary surface. As evident, in equation (24) the value $n_{j, m}$ vanishes when an element does not belong to the secondary surface.

The derivative of the nodal coordinates can be obtained by superposition of the source centre coordinates $C_{j}$ and the source local coordinates $\bar{X}_{j}$, i.e.,

$$
x_{j, m}=\left(C_{j}+\bar{X}_{j}\right)_{, m}=\bar{X}_{j, m}
$$

Hence, only the derivatives with respect to each of the three rotations in the fixed coordinate system are required. Finally, one obtains

$$
\begin{gathered}
\overline{\mathbf{X}}_{, \phi}=\mathbf{R}_{1, \phi}^{-1} \mathbf{R}_{2}^{-1} \mathbf{R}_{3}^{-1} \overline{\mathbf{x}}=\mathbf{R}_{1, \phi}^{-1} \mathbf{R}_{1}^{-1} \overline{\mathbf{X}} \\
\overline{\mathbf{X}}_{, \theta}=\mathbf{R}_{1}^{-1} \mathbf{R}_{2, \theta}^{-1} \mathbf{R}_{3}^{-1} \overline{\mathbf{x}}=\mathbf{R}_{1}^{-1} \mathbf{R}_{2, \theta}^{-1} \mathbf{R}_{2} \mathbf{R}_{1} \overline{\mathbf{X}} \\
\overline{\mathbf{X}}_{, \psi}=\mathbf{R}_{1}^{-1} \mathbf{R}_{2}^{-1} \mathbf{R}_{3, \psi}^{-1} \overline{\mathbf{x}}=\mathbf{R}_{1}^{-1} \mathbf{R}_{2}^{-1} \mathbf{R}_{3, \psi}^{-1} \mathbf{R}_{3} \mathbf{R}_{2} \mathbf{R}_{1} \overline{\mathbf{X}}
\end{gathered}
$$

where 


$$
\begin{aligned}
& \mathbf{R}_{1, \phi}^{-1}=\left[\begin{array}{ccc}
-\sin \phi & -\cos \phi & 0 \\
\cos \phi & -\sin \phi & 0 \\
0 & 0 & 0
\end{array}\right] \\
& \mathbf{R}_{2, \theta}^{-1}=\left[\begin{array}{cccc}
0 & 0 & 0 \\
0 & -\sin \theta & -\cos \theta \\
0 & \cos \theta & -\sin \theta
\end{array}\right] \\
& \mathbf{R}_{3, \psi}^{-1}=\left[\begin{array}{ccc}
-\sin \psi & -\cos \psi & 0 \\
\cos \psi & -\sin \psi & 0 \\
0 & 0 & 0
\end{array}\right]
\end{aligned}
$$

Similarly, the derivative of the normal $n$ with respect to the rotation design variables is obtained as follows

$$
\begin{gathered}
n_{, \phi}=\mathbf{R}_{1, \phi}^{-1} \mathbf{R}_{1}^{-1} n \\
n_{, \theta}=\mathbf{R}_{1}^{-1} \mathbf{R}_{2, \theta}^{-1} \mathbf{R}_{2} \mathbf{R}_{1} n \\
n_{, \psi}=\mathbf{R}_{1}^{-1} \mathbf{R}_{2}^{-1} \mathbf{R}_{3, \psi}^{-1} \mathbf{R}_{3} \mathbf{R}_{2} \mathbf{R}_{1} n
\end{gathered}
$$

Relations (26) and (28) can be written in a compact form as follows

$$
\overline{\mathbf{X}}_{, m}=\mathbf{T}_{, m}^{-1} \overline{\mathbf{x}}
$$

and

$$
\mathbf{n}_{, m}=\mathbf{T}_{, m}^{-1} \mathbf{n}
$$

since the derivative with respect to $m$ of the rotating system are zero.

\section{EQUATION ASSEMBLY}

The equation (12) can be collocated at the node of each element $(j=1, \ldots N)$ to give the following system matrix [36] 


$$
\mathrm{Hu}=\mathrm{Gq}+\mathrm{p}
$$

where $\mathbf{H}$ and $\mathbf{G}$ collect integrals of the fundamental solutions on the boundary elements, $\mathbf{u}$ and $\mathbf{q}$ collect potentials and fluxes on the boundary, respectively. The vector $\mathbf{p}$ is generated by the $N P$ sources within the domain $\Omega$.

The boundary conditions can be easily accounted in (31), to obtain the following system of algebraic equations

$$
\mathbf{A Y}=\mathbf{B J}+\mathbf{p}=\mathbf{F}+\mathbf{p}
$$

where $\mathbf{Y}$ is the vector containing the unknown boundary potentials and fluxes, $\mathbf{J}$ is a vector collecting the prescribed BCs, $\mathbf{A}$ and $\mathbf{B}$ are two non-symmetric and densely populated coefficient matrices composed by the columns of the matrices $\mathbf{H}$ and $\mathbf{G}$ that correspond to the unknowns and the prescribed BCs, respectively. Finally, $\mathbf{F}$ is obtained by multiplying the matrix $\mathbf{B}$ with the vector $\mathbf{J}$.

The collocation method can be also applied to (13) to give the following system of equations

$$
\mathbf{H}_{, m} \mathbf{u}+\mathbf{H} \mathbf{u}_{, m}=\mathbf{G}_{, m} \mathbf{q}+\mathbf{G q}_{, m}+\mathbf{p}_{, m}\left(\mathbf{X}^{\mathbf{s}}\right)
$$

where $\mathbf{H}, \mathbf{G}, \mathbf{u}$ and $\mathbf{q}$ are known after the solution of the direct problem (32), $\mathbf{H}_{, m}$ and $\mathbf{G}_{, m}$ have been outlined in section 4 , and $\mathbf{u}_{, m}$ and $\mathbf{q}_{, m}$ are the unknown sensitivities. The sensitivity boundary conditions are easily gathered from the boundary conditions of the direct problem, i.e., $\mathbf{u}_{m}=0$ at the boundary points where $\mathbf{u}$ is given or $\mathbf{q}_{, m}=0$ where $\mathbf{q}$ is assigned. Finally, $\mathbf{p}_{, m}$ is a vector created by the partial derivatives of the $N P$ sources within the domain $\Omega$ with respect to $m$. As seen in (31) and (32), equation (33) can be rearranged after imposing the boundary conditions, i.e.,

$$
\mathbf{A Y}_{, m}=\mathbf{A}_{, m} \mathbf{Y}+\mathbf{B}_{, m} \mathbf{J}+\mathbf{p}_{, m}
$$


As evident the term related to $\mathbf{B J}_{, m}$ is not included in the above system of equations since the derivative of the prescribed BCs with respect to the design variables $\left(\mathbf{J}_{, m}\right)$ is zero.

The potential and its derivative with respect to the design variable at the internal points are evaluated utilising equations (16) and (17). The internal point potential is given by

$$
\mathbf{U}=-\overline{\mathbf{H}} \mathbf{u}+\overline{\mathbf{G}} \mathbf{q}+\mathbf{P}
$$

where $\overline{\mathbf{H}}$ and $\overline{\mathbf{G}}$ involve integrals of the fundamental solution with source point coincident with any internal point and $\mathbf{P}$ refers to the effects of the $N P$ extra sources. The vector $\mathbf{U}_{, m}$ of the potential derivatives at the internal points is written as follows

$$
\mathbf{U}_{, m}=-\overline{\mathbf{H}} \mathbf{u}_{, m}-\overline{\mathbf{H}}_{, m} \mathbf{u}+\overline{\mathbf{G}}_{\mathbf{q}_{, m}}+\overline{\mathbf{G}}_{, m} \mathbf{q}+\mathbf{P}_{, m}
$$

Equations (35) and (36) provide both the terms involved in the cost function (2) and its derivative (9) with respect to $\mathbf{a}$ or $\mathbf{b}$.

In general, the procedure that yields the optimum secondary source location and orientation requires at every iteration the calculation of the matrices involved in equations (31-36) and the resolution of the two systems of equations (32) and (34). In fact, the secondary source is part of the geometry of the problem and a variation of its location modifies the solution of both the primary and the secondary fields. On the contrary, the optimum CV location depends only upon the position of the $\mathrm{CV}$ points and, therefore, the boundary solution of the direct problem does not need to be updated and the boundary sensitivities are zero.

\section{NUMERICAL ASPECTS}

\subsection{Matrix Update.}

In this paragraph some implementation aspects of the formulation for the optimum secondary source location/orientation are detailed. 
The two systems of equations (32) and (34) are different at each secondary source location, hence at each iteration they are assembled and solved twice, for the primary and for the secondary fields (as evident, in the case of optimum control source rotation the primary field is evaluated only once). Nevertheless, not all the coefficients of the matrices $\mathbf{H}, \mathbf{G}, \mathbf{H}_{, m}$, and $\mathbf{G}_{, m}$ are calculated at each step.

Let us consider that the element coefficients referred to the secondary source are located at the beginning of the matrices and those referred to the remaining boundary of the domain at the end. Hence the matrices $\mathbf{H}, \mathbf{G}, \mathbf{H}_{, m}$, and $\mathbf{G}_{, m}$ can be divided into four parts as shown in figure 5 on the basis of the position of the source points and of the integration elements.

The $\mathbf{M}_{\mathbf{i i}}$ and $\mathbf{M}_{\mathbf{i j}}$ parts of figure 5 refer to the effect on the secondary source generated by itself and by the remaining part of the boundary, respectively, while the $\mathbf{M}_{\mathbf{j i}}$ and $\mathbf{M}_{\mathbf{j} \mathbf{j}}$ parts collect the integrals when the source point is placed anywhere except on the secondary source and the integration element moves both on the secondary source $\left(\mathbf{M}_{\mathbf{j i}}\right)$ and on the remaining boundary $\left(\mathbf{M}_{\mathbf{j} \mathbf{j}}\right)$.

As evident, $\mathbf{M}_{\mathbf{i i}}$ and $\mathbf{M}_{\mathbf{j} \mathbf{j}}$ remain unchanged for a new secondary source location/orientation, whereas the matrices $\mathbf{H}_{, m}$, and $\mathbf{G}_{, m}$ have zero coefficients in such portions. Hence, at each iteration the $\mathbf{H}, \mathbf{G}, \mathbf{H}_{, m}$, and $\mathbf{G}_{, m}$ matrices are evaluated only at the $\mathbf{M}_{\mathbf{j i}}$ and $\mathbf{M}_{\mathbf{i j}}$ portions for both the primary and secondary fields.

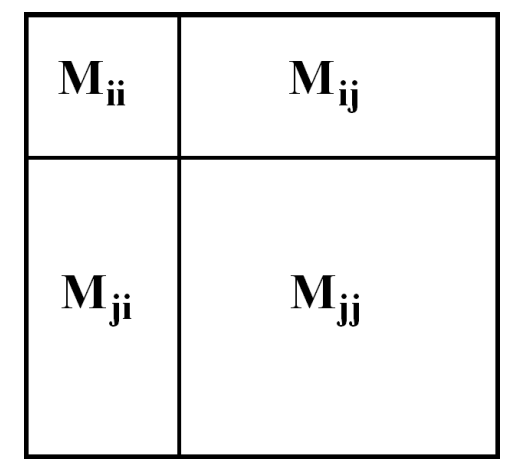

Figure 5. Matrix division.

\subsection{Rapid Solver.}


Due to the fact that the BEM system matrix is non-symmetric and fully populated, the solutions of the two systems (32) and (34) are time consuming, especially in optimisation and identification problems where the solution is required many times. Moreover, each term of the cost function (2) and its derivative (9) requires the evaluation of the internal point quantities given by the equations (35) and (36); such a task can considerably increase the solution time in the case of large sized CVs.

In the recent past, various techniques have been explored to accelerate the BEM solution time, for instance block-based solvers [41], lumping techniques [42], iterative solvers [43], fast multiple method [44]. In this paper, the Adaptive Cross Approximation (ACA) in conjunction with the $\mathcal{H}$-matrix format and the iterative solver GMRES is utilised to accelerate the CPU time. The ACA is a pure algebraic technique widely investigated [45] and applied in many engineering fields [46] among which the Helmholtz equation [21]. The solving matrix is divided into two groups of blocks, full - rank and low - rank blocks. The former blocks are calculated entirely, while the latter blocks are replaced by few entries.

An admissible low-rank block $C_{a}$ can be substituted by an approximated block $C_{\bar{k}}$ as follows

$$
C_{a} \simeq C_{\bar{k}}=\sum_{i=1}^{\bar{k}} a_{i} \cdot b_{i}^{T}
$$

where $a_{i}$ and $b_{i}$ denote the columns and the rows, respectively, of the approximated block. The approximating block $C_{\bar{k}}$ satisfies the relation $\left\|C_{a}-C_{\bar{k}}\right\|_{F} \leq \varepsilon\left\|C_{a}\right\|_{F}$, where $\|\cdot\|_{F}$ represents the Frobenius norm and $\varepsilon$ is the prescribed accuracy. It should be noted that the solution obtained is adaptively approximated and maintains a required level of accuracy; a higher accuracy level is adaptively reached by a higher value of $\bar{k}$. The value $\bar{k}$ is, in general, much less than the rank of the original block. This permits to speed up the assembly time, the storage requirements as well as the matrix-vector product at every iteration.

The admissibility condition that leads the existence of a low-rank block is written as follows 


$$
\min \left(\operatorname{diam} \Omega_{x_{0}}, \operatorname{diam} \Omega_{x}\right) \leq \varrho \cdot \operatorname{dist}\left(\Omega_{x_{0}}, \Omega_{x}\right)
$$

where $\Omega_{x_{0}}$ and $\Omega_{x}$ denote the cluster of the elements corresponding to the row and column indices of the considered block, respectively, $\varrho$ is a non-zero parameter that influences both the number of admissible blocks and the convergence speed of the adaptive approximation of low rank blocks $[45,46]$.

The idea behind this technique is based on the consideration that the integrals of contiguous elements due to a single collocation point are similar, especially for high density meshes. The same consideration is valid for the integrals of a single element due to a number of contiguous collocation points.

The main advantage of the ACA is that the kernel is not substituted (as happens in the Fast Multiple Method) and only few entries are required, therefore most part of existing BEM codes can be used without meaningful changes.

The proposed method has been applied to accelerate both the assembly time of matrices $\mathbf{H}$ and $\mathbf{G}$, and the post-processing step (aimed at calculating the potential of the internal points that constitute the CV) of matrices $\overline{\mathbf{H}}$ and $\overline{\mathbf{G}}$. Furthermore, since the singularities of the kernels involved in the sensitivity problem are of the same order as the singularities of the kernels involved in the direct approach [24], the sensitivity matrices, $\mathbf{H}_{, m}$ and $\mathbf{G}_{, m}$ of equation (33) and $\overline{\mathbf{H}}_{, m}$ and $\overline{\mathbf{G}}_{, m}$ of equation (36), can all be evaluated using the proposed approach. It is worth noting that the programming effort for the application of the $\mathcal{H}$-matrix $\mathrm{ACA}$ is the same for the two systems of equations (i.e., the direct system and the sensitivity system).

Finally, it should be pointed out that the computational cost associated with evaluating the cost function and its gradients (once the relative quantities at any internal points of the CV are computed) is negligible compared with the overall solution time.

Comparisons between the proposed strategy and the standard procedure can be found in many papers (see for instance $[21,45,46]$ ). 
Figure 6 shows a solving matrix representation after the application of the ACA $(\varrho=50)$ to an aircraft cabin composed by 8000 degrees of freedom (see figure 16). The dark gray and the light gray block represent the full rank and the low rank blocks, respectively. In particular, the more the light gray, the lower the value of the rank $\bar{k}$ of the blocks. As evident almost the whole matrix is represented in a low rank format.

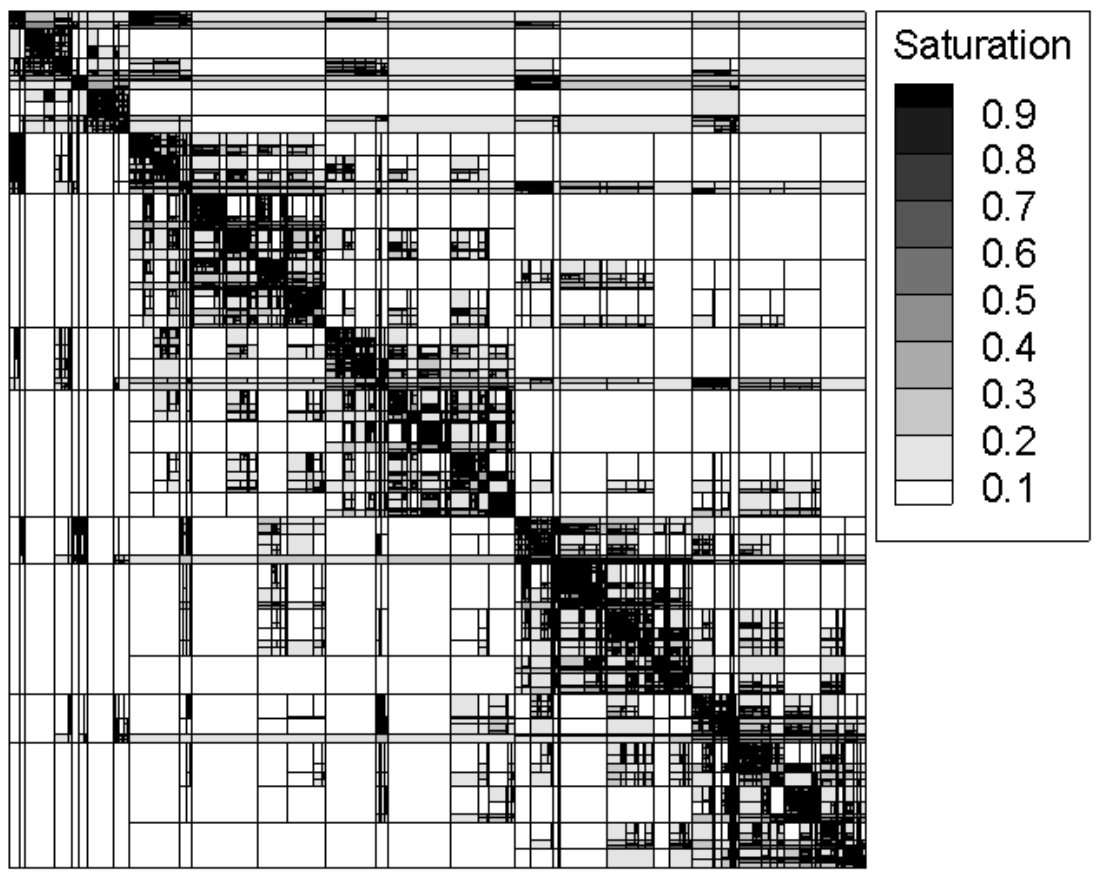

FiguRE 6. Block-wise representation of an ACA generated matrix.

\section{NUMERICAL EXAMPLES}

In this section four different examples are presented to test the proposed procedure. The secondary field is created by a hard $(q=0)$ spherical speaker, with radius equal to $4 \mathrm{~cm}$ and with a $120^{\circ}$ vibrating segment (with constant $q$ ), which is meshed with 120 super-parametric (linear geometry, constant unknown) elements in the first example and 224 elements in the other examples (see figure 7). 
The optimum secondary source location is obtained in terms of the coordinates of the sphere's centre.

The noise reduction is calculated as suggested by Bermudez et al. [47], i.e.,

$$
\operatorname{Attenuation}\left(\mathrm{X}^{\prime}\right)=-10 \log _{10}\left(\frac{f_{c}\left(\mathrm{X}^{\prime}\right)_{o p}}{f_{c}\left(\mathrm{X}^{\prime}\right)_{0}}\right)
$$

where $f_{c}\left(\mathrm{X}^{\prime}\right)_{o p}$ and $f_{c}\left(\mathrm{X}^{\prime}\right)_{0}$ are the values at the internal points $\mathrm{X}^{\prime}$ of the optimized cost function (2) and of the cost function either without active control or with a not optimised location/orientation, respectively.

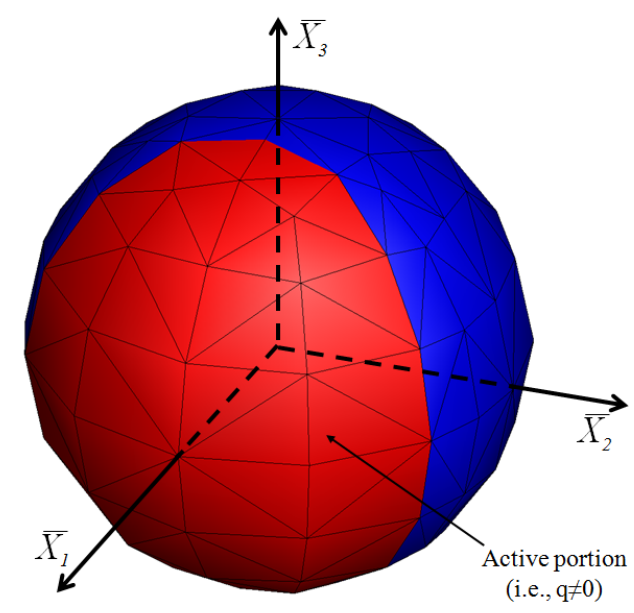

FiguRE 7 . Secondary source modelled as a rigid sphere with a $120^{\circ}$ active segment.

The integral over the CV is approximated using 8 node brick cells and then evaluated by the Legendre-Gauss quadrature rule.

The geometry of the second example has been taken from ref. [19].

For internal problems the optimisation routine (in ref. [40]) has been constrained to avoid the secondary source going outside the domain along the iteration path. The four numerical examples are summarized in table 2. 


\begin{tabular}{c|c|c}
\hline Optimisation procedure & Example & Geometry \\
\hline CV location & $1^{\text {st }}$ & Unbounded domain \\
\hline Secondary source location & $2^{\text {nd }}$ & $4 m \times 3 \mathrm{~m} \times 2.5 \mathrm{~m}$ room \\
\hline Secondary source orientation & $3^{\text {rd }}$ & $1 \mathrm{~m} \times 0.5 \mathrm{~m} \times 0.5 \mathrm{~m}$ box \\
\hline Secondary source location/orientation & $4^{\text {st }}$ & Airplane cabin \\
\hline
\end{tabular}

TABLe 2. Numerical examples.

\subsection{Optimum Location.}

The first example concerns the location of the optimum CV in a confined region close to the secondary source.

A diffuse noise is generated in a $1 \times 1 \mathrm{~m}^{2}$ plane to simulate the effects of counteracting waves bouncing off the walls in the proximity of the secondary source in a possible local ANC configuration. The analysis is then focused on the same plane and all the possible CV locations are permitted only in this area.

The primary noise potential at each point of coordinate $\mathbf{X}$ is given by $[7,8]$

$$
U_{p}(\mathbf{X})=\sum_{j=1}^{j_{\max } l_{\max }} \sum_{l=1}\left(a_{j l}-i b_{j l}\right) \mathbf{e}^{i k \hat{e}_{k} \cdot \mathbf{X}}
$$

where $k$ is the wave-number, $\hat{e}_{k}$ is the plane wave versor and $\cdot$ is the scalar product operator. In order to create a diffuse primary noise, 72 plane waves distributed in all directions of the space are generated with $j_{\max }=6$ and $l_{\max }=12$. The values of $a_{j l}$ and $b_{j l}$ are randomly chosen from a uniform distribution. The origin of the global coordinate system coincides with the sphere centre. The CV is $10 \times 16 \times 8$ $\mathrm{cm}^{3}$ and it has been meshed by 140 nodes and 72 8-nodes brick elements.

Figure 8 shows the variation of the cost function versus the $x_{1}$-coordinate of the CV centre for four different frequencies $(109,273,546,1092 \mathrm{~Hz})$ and within 1.845 $m$ from the secondary source centre. It can be noted that the higher the frequency, the higher the number of local minima and the lower the noise attenuation level. 
As evident, from figure 8, some local minima may occur and a unique optimum CV location for all frequencies cannot be evaluated.

Figure 9 shows for each frequency the total (primary and secondary fields) potential amplitude distribution for one local optimum value (a trough in figure 8).

Such results are discussed in the appendix.

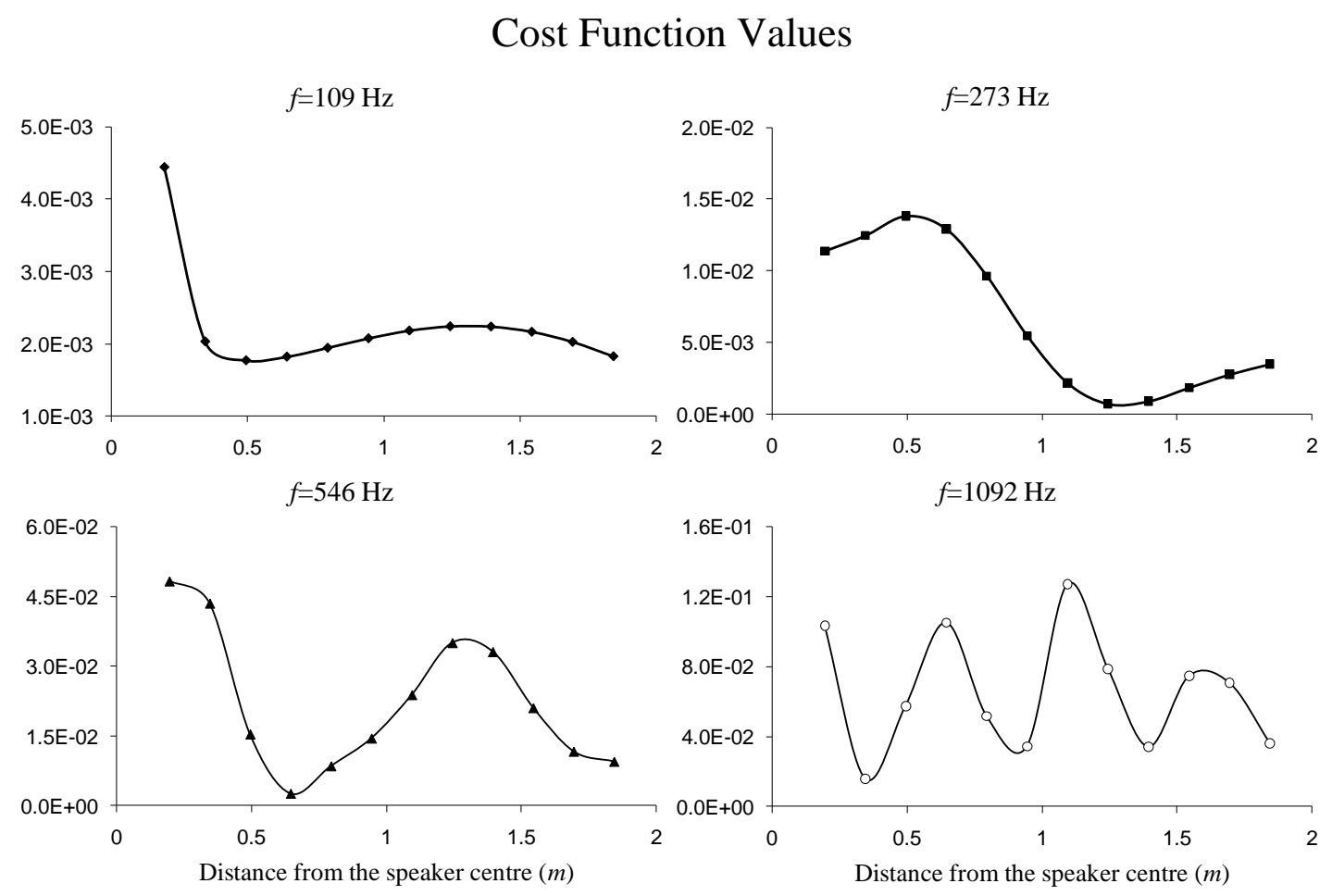

Figure 8. The cost function $f_{c}$ for four different frequencies. (Example 1)

\subsection{Optimisation of the Secondary Source Location.}

In the second example the location of the secondary speaker is optimized inside a room with dimensions (in metres) $4 \times 3 \times 2.5$ that is discretized by 1512 nodes 

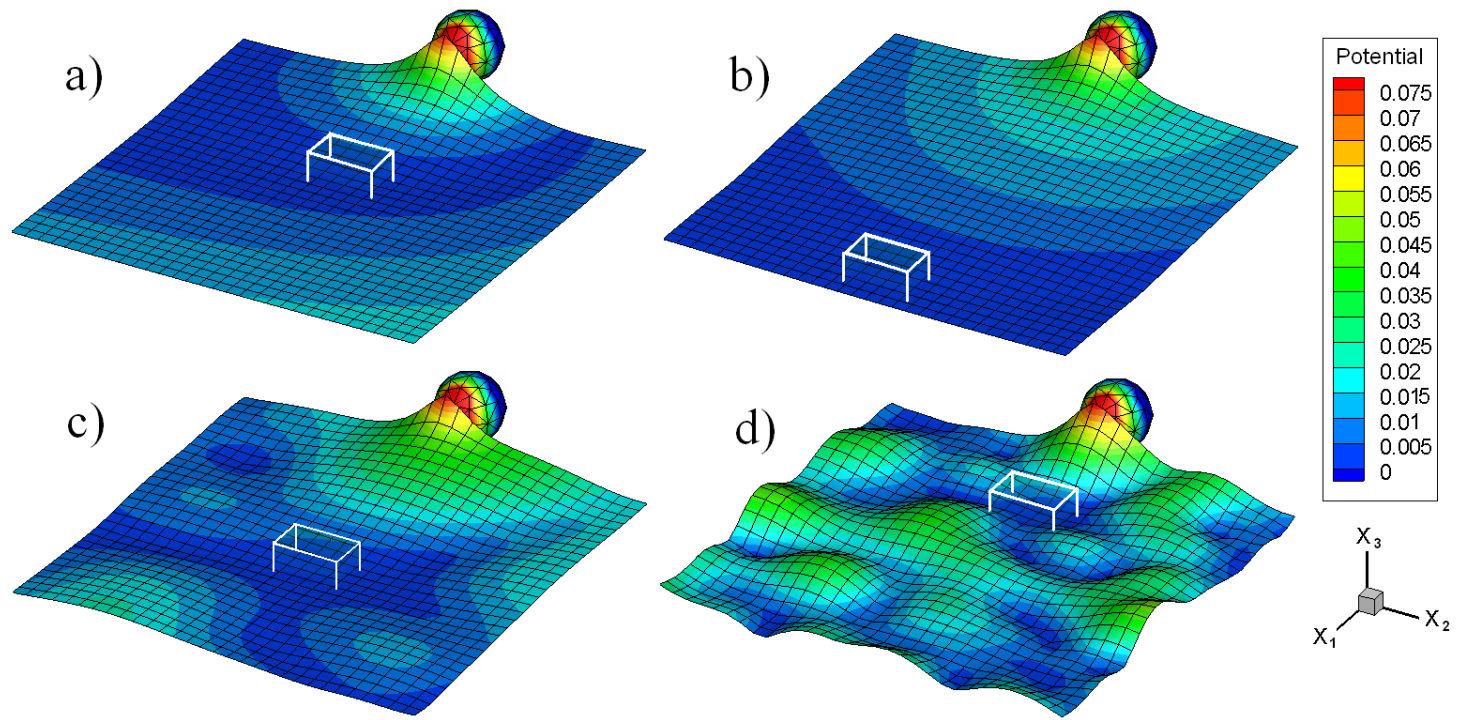

Figure 9. Total amplitude potential for the optimum $\mathrm{CV} x_{1^{-}}$ locations corresponding to the trough of the cost function in figure 8: a) $109 \mathrm{~Hz}$; b) $273 \mathrm{~Hz}$; c) $546 \mathrm{~Hz}$; d) $1092 \mathrm{~Hz}$. (Example 1)

and 3020 super-parametric (linear geometry, constant unknowns) elements. The walls are modelled as hard, hence the sound is not absorbed. The primary noise has unitary amplitude and it is generated by a vibrating surface placed from (2.6, $3.0,2.0)$ to $(3.0,3.0,2.2)$ in one of the walls, i.e., a noisy air gate is simulated. The reduction of the noise is requested in a cubic CV with opposite vertexes $(0.5,0.5,0.8)$ - $(2.5,1.5,2.0)$, composed by 2640 point and 2090 linear brick elements. The frequency is $78 \mathrm{~Hz}$ close to the first resonance frequency $(85 \mathrm{~Hz})$. The secondary source centre is initially located at the point $(3.6,2.6,0.0)$ and its vibrating surface is orientated along the positive $x_{1}$-axes (initial guess). At this position the cost function value is $f_{c}=2.01910^{-2}$. The geometry of the problem is depicted in figure 10, where the room, the CV and the vibrating surface of the wall are highlighted.

The centre location of optimum secondary source is at $(2.615,2.587,0.105)$ where the cost function value is $f_{c}=1.52910^{-4}$. The noise attenuation obtained 
by the optimisation process is $21.21 \mathrm{~dB}$. This confirms that a correct location of the secondary source can greatly influence the local ANC performances.

Figures 11 show the sound pressure level (SPL) contour plot in the whole room and inside the $\mathrm{CV}$ for the initial guess (a) and for the final optimum location (b). The surface translucency of the room walls has been increased to permit a better visualisation of the SPL distribution inside the CV. The initial and final location of the secondary source can be easily distinguished. It should be noted that the indicated SPL has not any physical meaning and it is just the result of the simulation with the boundary conditions described above.

In figure 12 the cost function trend variation against the number of iterations is presented.

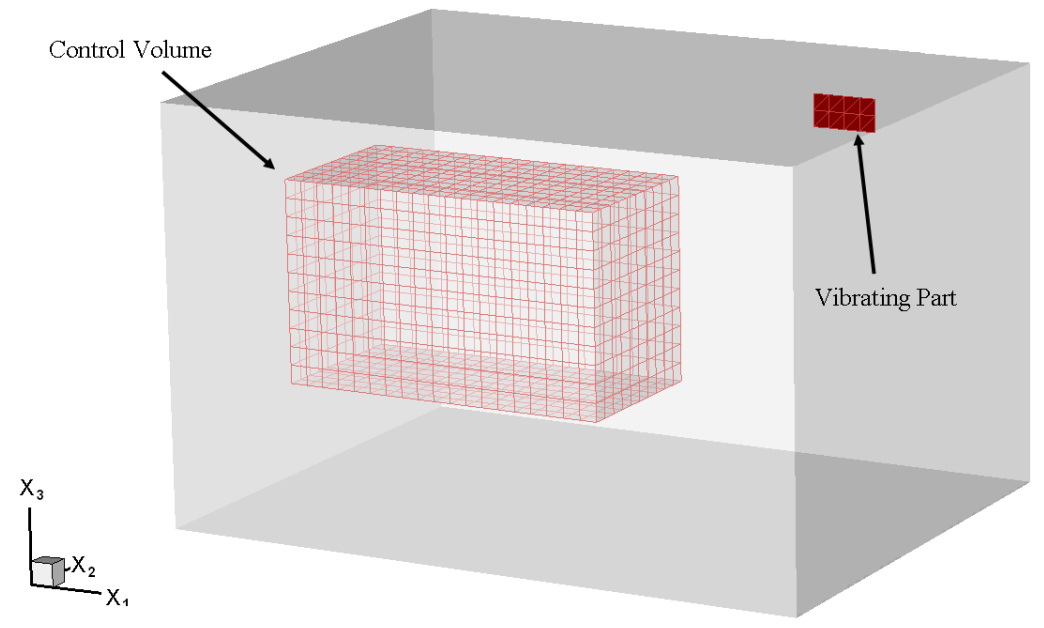

Figure 10. Room geometry, CV and primary noise locations. (Example 2)

\subsection{Optimisation of the Secondary Source Orientation.}

This example (see figure 13) concerns the optimal orientation of the secondary source inside a box with dimensions $1 \times 0.5 \times 0.5$ (in metres). The noise attenuation 

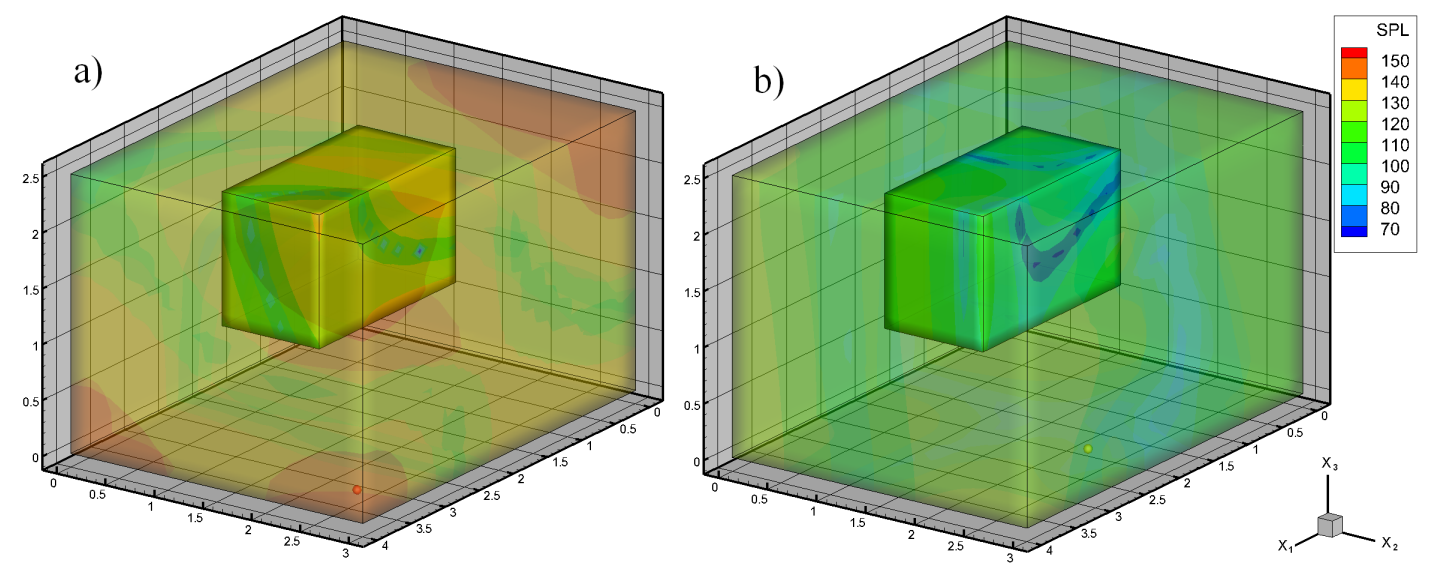

Figure 11. SPL distribution (dB) inside a $4 \times 3 \times 2.5 \mathrm{~m}$ room generated by the application of the ANC: a) not optimised and b) optimised secondary source location. (Example 2)

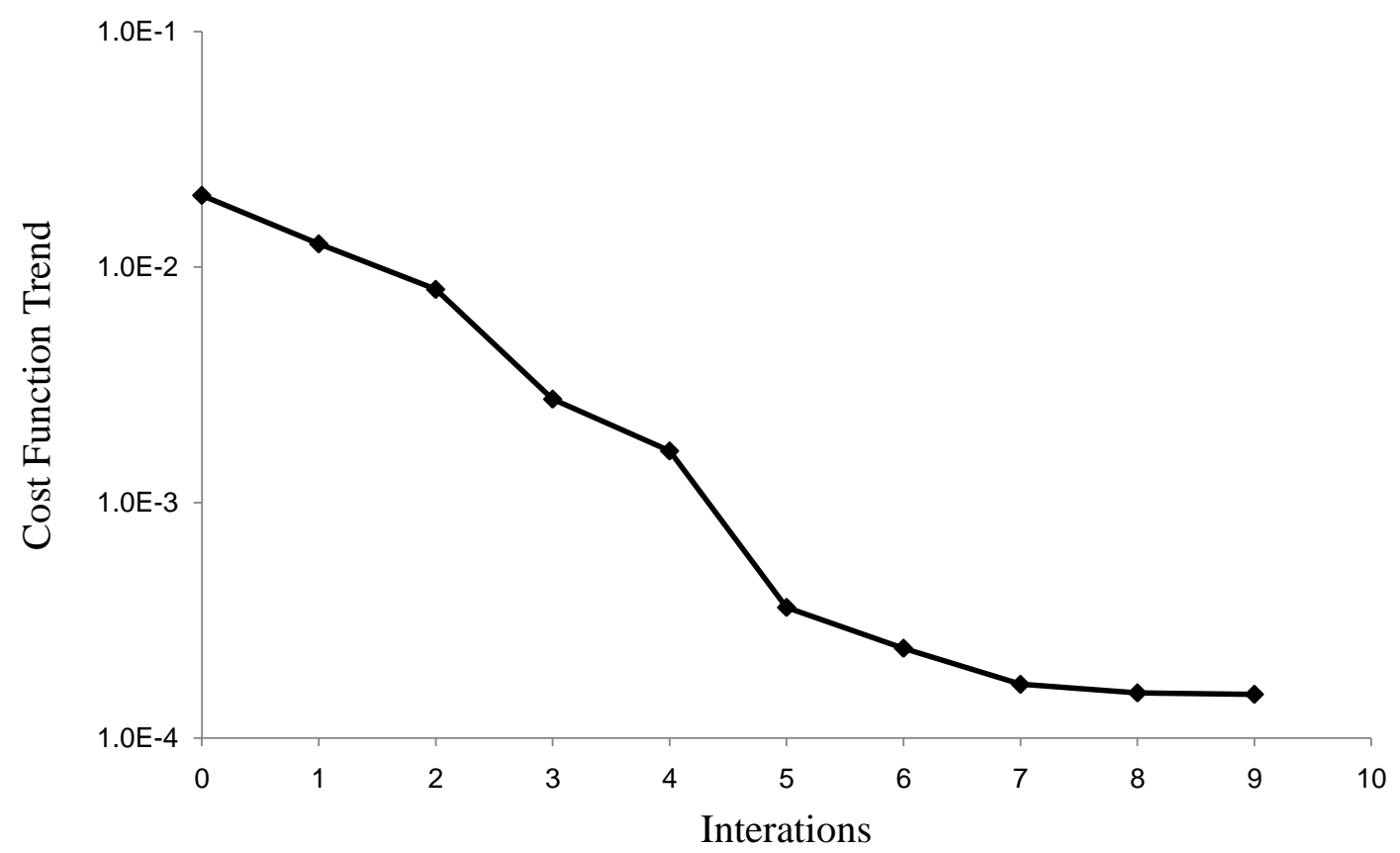

Figure 12. Cost function trend variation with number of iterations. (Example 2) 
is requested in a small cuboid $\mathrm{CV}(0.1 \times 0.05 \times 0.05 \mathrm{~m})$, with the two opposite corners at $(0.7,0.3,0.3)$ and $(0.8,0.35,0.35)$.

The box is meshed by 1002 nodes and 2000 super-parametric (linear geometry, constant unknown) elements. The integration in the CV is performed by subdividing it into 16 cuboid linear elements. The primary noise has unitary amplitude and it is generated by a vibrating portion of a wall of the box, with opposite corners at $(0.75,0.25,0.5)$ and $(0.85,0.4,0.5)$ (see figure 13$)$. The remaining walls are modelled as hard $(q=0)$. The frequency chosen is $335 \mathrm{~Hz}$, close to the first resonance frequency $(340 \mathrm{~Hz})$. The centre of the secondary source is located at the point $(0.075,0.075,0.425)$ and its vibrating surface is orientated along the negative $x_{2}$-axes (initial guess). At this position the cost function value is $f_{c}=5.16310^{-5}$. The geometry of the problem is depicted in figure 13, where the $\mathrm{CV}$, the secondary source and its vibrating segment, as well as the vibrating surface of the wall can be easily distinguished.

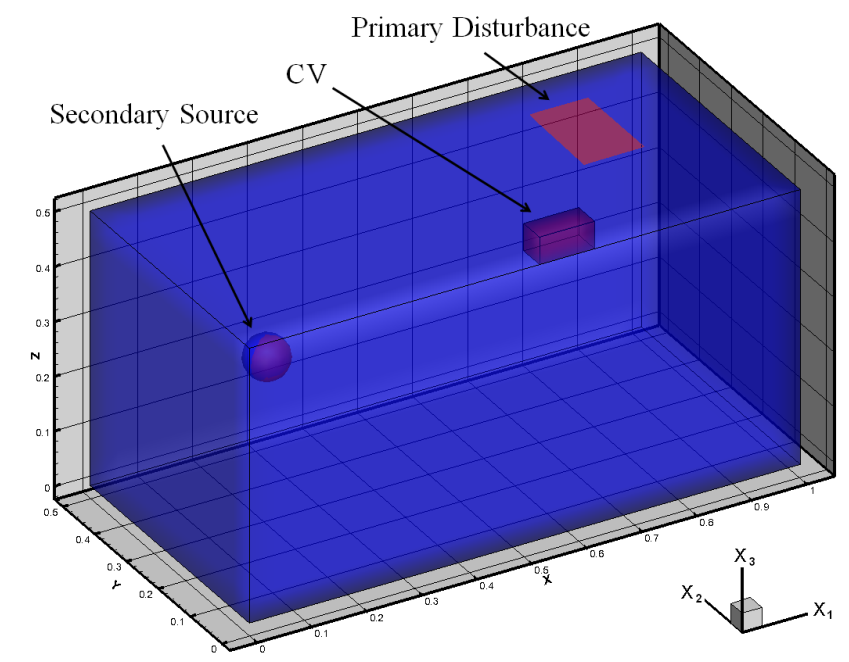

FiguRE 13. Geometry of the problem, CV, control source (initial guess) and primary noise locations. (Example 3)

In figure 14 the SPL contour plot in the whole box for two different secondary source orientations (i.e., a - initial guess $(\phi=0, \theta=0, \psi=0)$; and b - optimal 
configuration $(\phi=0.305, \theta=0.857, \psi=0.092))$ is shown. The optimum cost function value is $f_{c}=1.57610^{-5}$ and the obtained noise attenuation amounts to $5.15 \mathrm{~dB}$.

In figure 15 a zoom on the initial guess and on the final secondary source orientation is depicted.
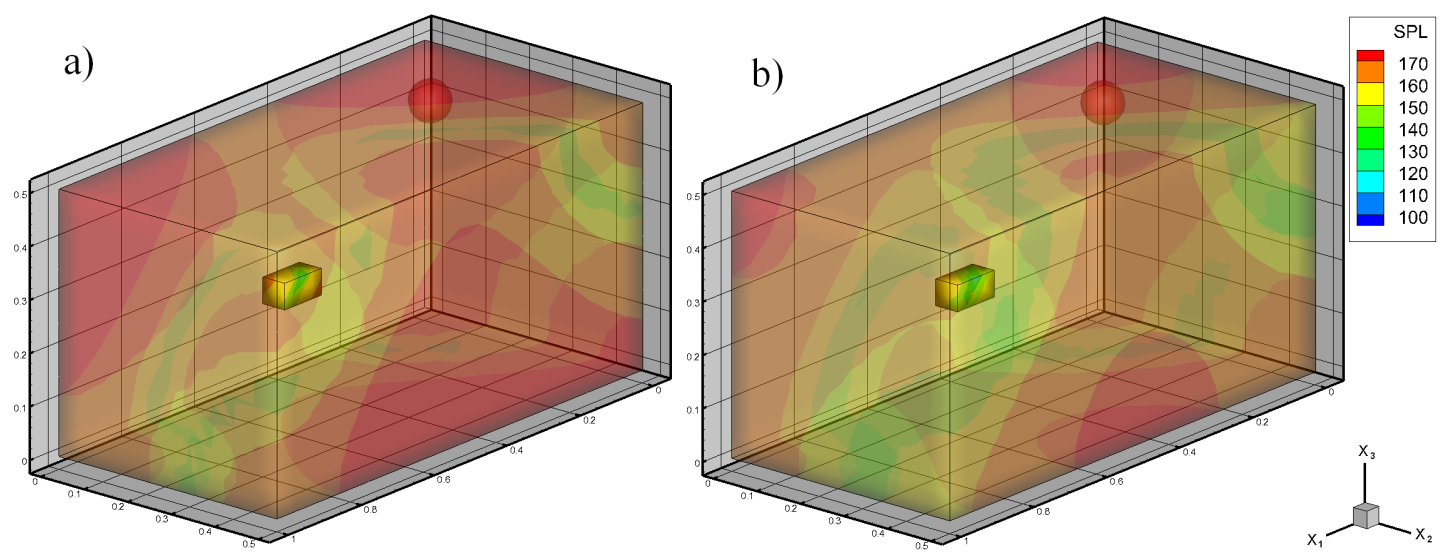

FiguRE 14. SPL distribution (dB) inside the box of the total field generated by the ANC application a) initial guess, and b) optimal value. (Example 3)

\subsection{Optimisation of the Secondary Source Location in a Large-Scale Engineering Problem.}

This simulation concerns the evaluation of the optimum location/orientation of a secondary source inside an airplane cabin.

The model of the analysed cabin consists of two lines of three seats surrounded by the aircraft fuselage. Due to the geometrical symmetry, only one half of the cabin is considered.

The cabin is included in a cuboid of dimensions $2 \times 1.9 \times 2.3$ (in metres) $m$ and each line of seats has dimensions $0.5 \times 1.35$ with height 1.054 . The headrest is 0.46 long (along the $x_{2}$-axes) and the $\mathrm{CV}$ is located in the middle front seat and it has 

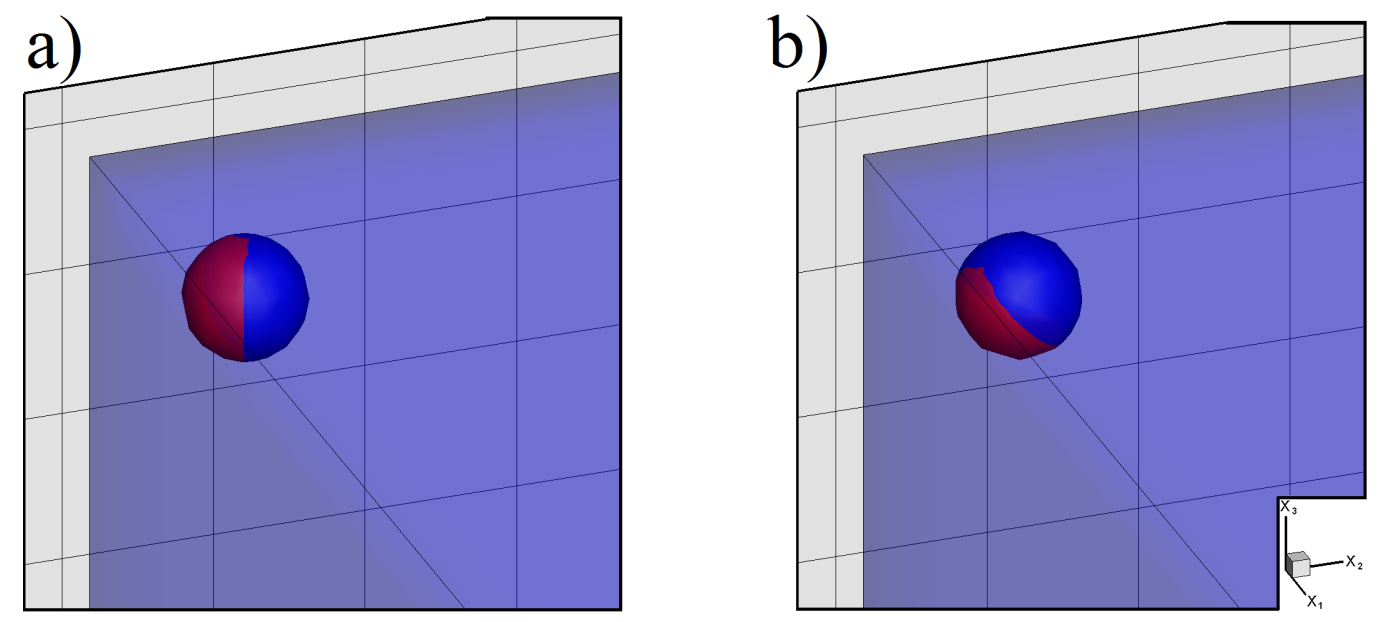

FiguRE 15. Zoom on the secondary source: a) initial guess, and b) optimal value. (Example 3)

dimensions $0.2 \times 0.32 \times 0.2$, therefore it covers most of the headrest extension. The cabin is meshed by 3914 nodes and 7699 constant elements in order to deal with up to $250 \mathrm{~Hz}$ (i.e., 10 elements per wavelength are guaranteed). The integration in the $\mathrm{CV}$ is performed by subdividing it into 160 cuboid linear elements.

The boundary conditions have been set in order to simulate a real case, hence the rear and front panel are modelled as soft surfaces $(u=0)$. The remaining surfaces, i.e., seats, floor, ceiling, have all been modelled with absorbing coefficients being $0.85,0.2$ and 0.15 . In order to evaluate the impedance value, the impedance phase is set to zero [48].

The noise source is generated by a monopole source located at the point (1.46, $0.52,0.87)$ in front of the rear seat close to the symmetry panel, whereas the secondary field is modeled as a rigid sphere (centered in $(0.76,1.02,1.46)$ ) with an active segment orientated along the negative $x_{3}$-axes.

In the figures 16 and 17 the model representing the aircraft cabin, the primary monopole source, the $\mathrm{CV}$ and the secondary source are depicted (where the symmetry panel has been removed for a better view of the model).

Initially, a series of simulations were performed to evaluate the resonance frequencies of the cabin. The cabin response (in terms of potential) of a typical jet 
with a noise frequency range $(70-200 \mathrm{~Hz})$ [49] is studied and the cabin response at a point located away from the anti-node points is presented in figure 18. As evident from the figure there are only two picks, hence the simulations were performed only for two frequencies (104 and $182 \mathrm{~Hz}$ ).

In the first simulation the final location of the control source centre is at the point $\left(8.57610^{-1}, 5.18210^{-1}, 1.641\right)$, whereas the vibrating portion is slightly modified by the procedure $\left(-9.27910^{-4},-2.05810^{-2}, 6.11310^{-3}\right)$. The initial and the final cost function values are $f_{c}=2.89510^{-3}$ and $f_{c}=4.01510^{-4}$, respectively. Hence, the optimisation procedure is able to reduce the noise level of $8.58 \mathrm{~dB}$.

At $182 \mathrm{~Hz}$ the optimisation procedure has a lower performance as the noise level from the original configuration is reduced by only $1.33 \mathrm{~dB}$, and the optimal location and orientation of the secondary source are $\left(7.19510^{-1}, 1.085,1.558\right)$ and $\left(1.44610^{-4}, 5.13310^{-2},-2.90810^{-3}\right)$, respectively.

However, the presence of the secondary source in its optimised configuration reduces the noise levels of $22.49 \mathrm{~dB}$ and $11.75 \mathrm{~dB}$ at $104 \mathrm{~Hz}$ and $182 \mathrm{~Hz}$, respectively.

In figure 19 the total SPL generated inside the cabin at $104 \mathrm{~Hz}$ by the initial guess (a) and by the optimum location/orientation (b), are depicted. In the same figure it can be also noted that the control source is able to reduce the global primary noise, even though this is just a side effect.

\section{CONCLUSIONS}

In this paper a sensitivity analysis for a local ANC approach was developed. The 3D acoustic propagation problem was modelled by the Helmholtz equation and numerically solved by coupling the BEM, with the ACA, the $\mathcal{H}$-matrix format and the GMRES solver. The optimisation procedure was performed by applying a first order Quasi Newton method. The sensitivities were determined by the implicit 


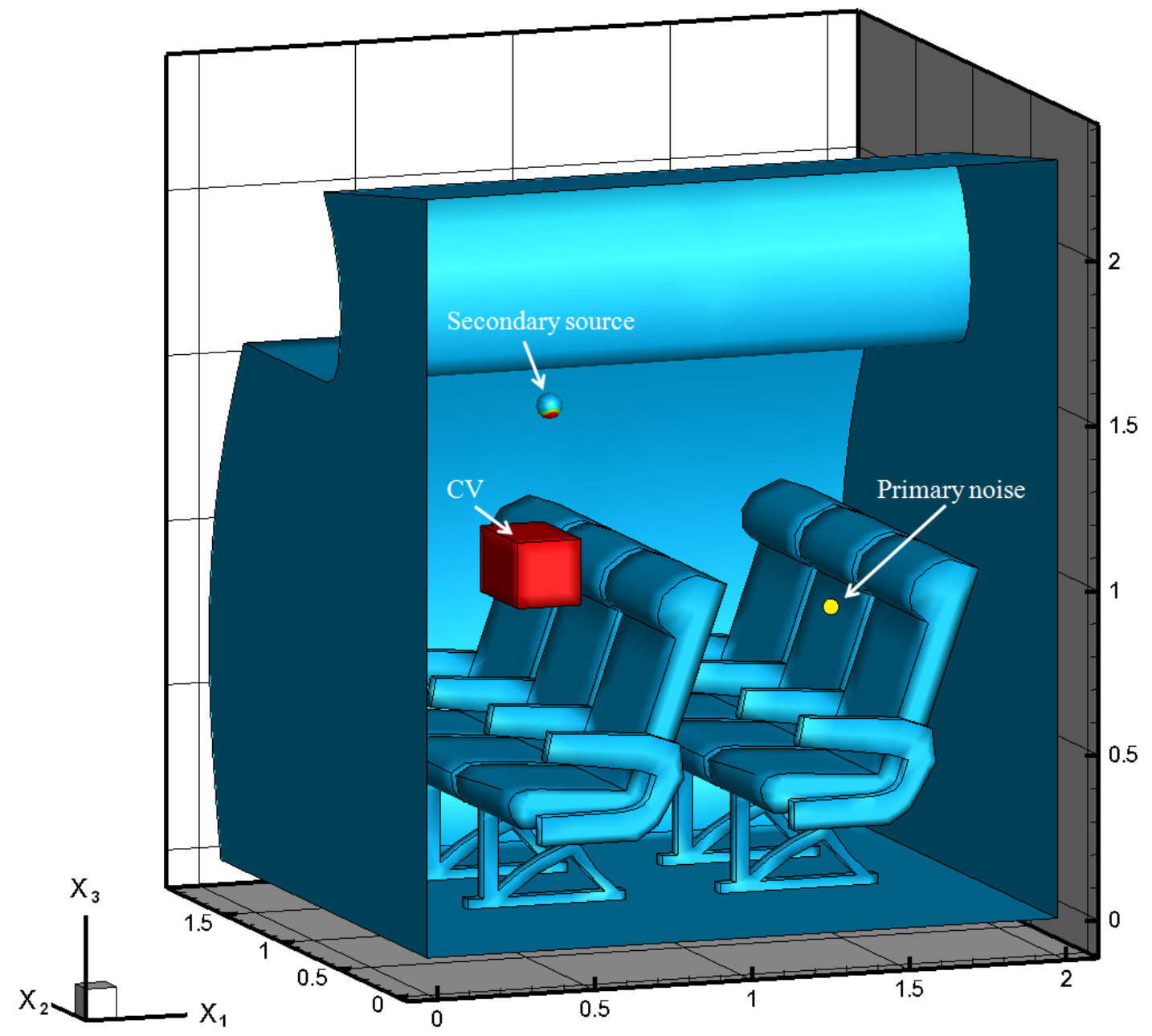

FIGURE 16. Geometry of the cabin: CV, initial secondary source and primary noise locations. (Example 4)

differentiation approach. The noise reduction was achieved by attenuating the unwanted primary sound inside a confined volume, the CV, with the aid of a secondary acoustic source. The optimisation procedure was developed for both the optimum location of the $\mathrm{CV}$, and the optimum location and orientation of the secondary source; it is valid for any shape of both CV and control source.

Four examples were presented in order to demonstrate the efficiency of the procedure. The first referred to the optimum location of the CV, the second and the 


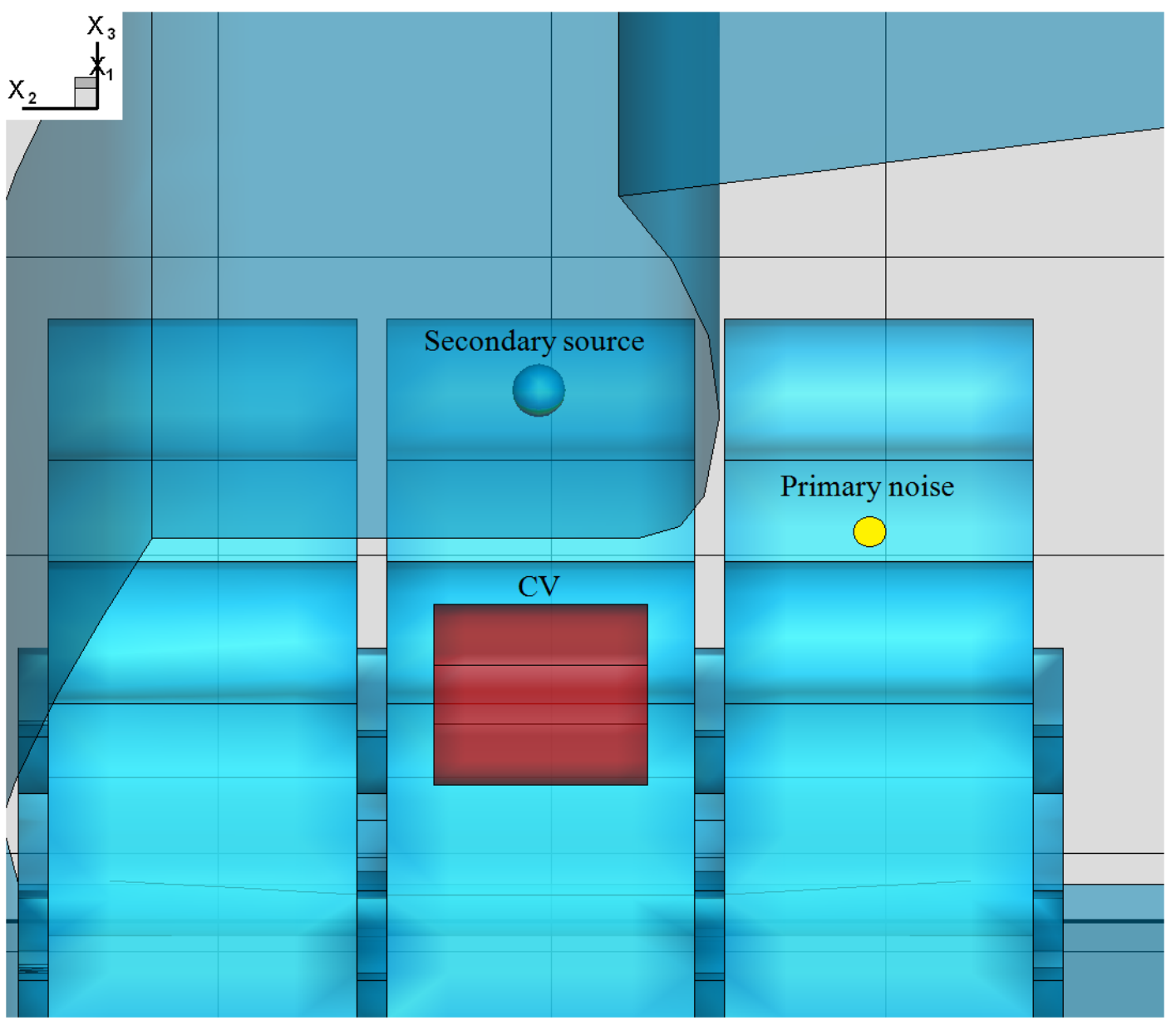

FiguRE 17. Zoom of the seat of the cabin: secondary source, CV and monopole source. (Example 4)

third found the optimum secondary source location and orientation, respectively; a forth example, a large-scale engineering problem (i.e., the best location and orientation of the loudspeaker for a local ANC strategy inside an aircraft cabin) was investigated.

Acknowledgments. This work was carried out with the support of European research project (SEAT: Smart tEchnologies for stress free Air Travel) AST5-CT2006-030958. 


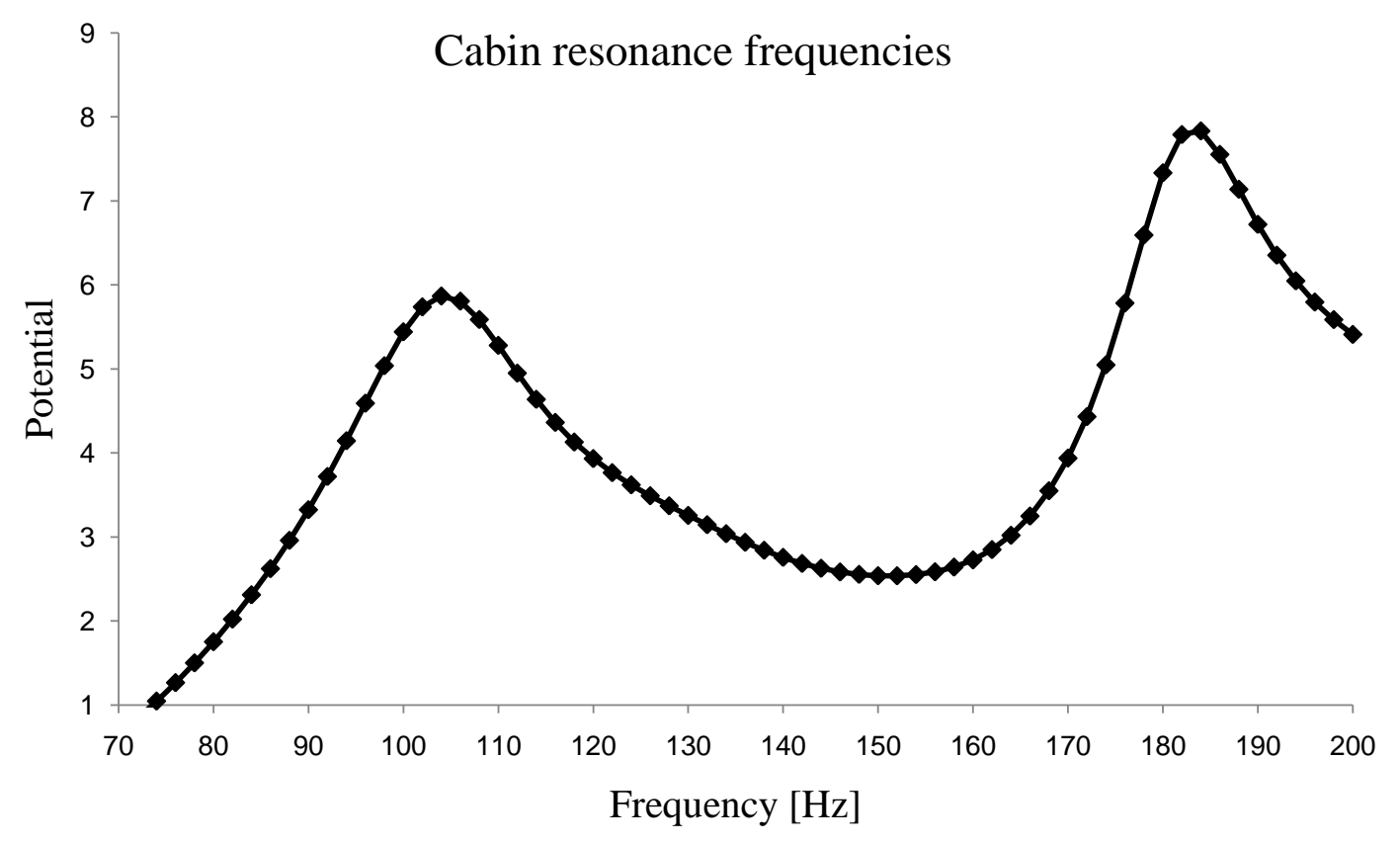

Figure 18. Potential over the frequency of the cabin under described boundary conditions. (Example 4)

\section{REFERENCES}

[1] Conover WB. Fighting noise with noise. Noise Control 1956; 2, pp.78-82.

[2] Nelson PA, Curtis ARD, Elliott SJ, Bullmore AJ. The active minimisation of harmonic enclosed sound fields: part i, ii, iii. Journal of Sound and Vibration 1987; 117(1), pp.158, DOI: 10.1016/0022-460X(87)90432-9, 10.1016/0022-460X(87)90433-0, 10.1016/0022460X(87)90434-2.

[3] Nelson PA, Curtis ARD, Elliott SJ, Bullmore AJ. The Minimum Power Output of Free Field Point Sources and The Active Control of Sound, Journal of Sound and Vibration 1987; 116, pp.397-414, DOI: 10.1016/S0022-460X(87)81373-1.

[4] Ross CF. Experiments on the active control of transformer noise. Journal of Sound and Vibration 1978; 61(4), pp. 473-480, DOI: 10.1016/0022-460X(78)90449-2.

[5] Hesselman N. Investigation of noise reduction in a $100 \mathrm{KVA}$ transformer tank by means of active methods. Applied Acoustics 1978; 11, pp. 27-34, DOI: 10.1016/0003-682X(78)900191.

[6] Berge T, Pettersen O, Sorsdal S. Active cancellation of transformer noise: Field measurements. Applied Acoustics 1988; 23, pp. 309-320, DOI: 10.1016/0003-682X(88)90044-8. 

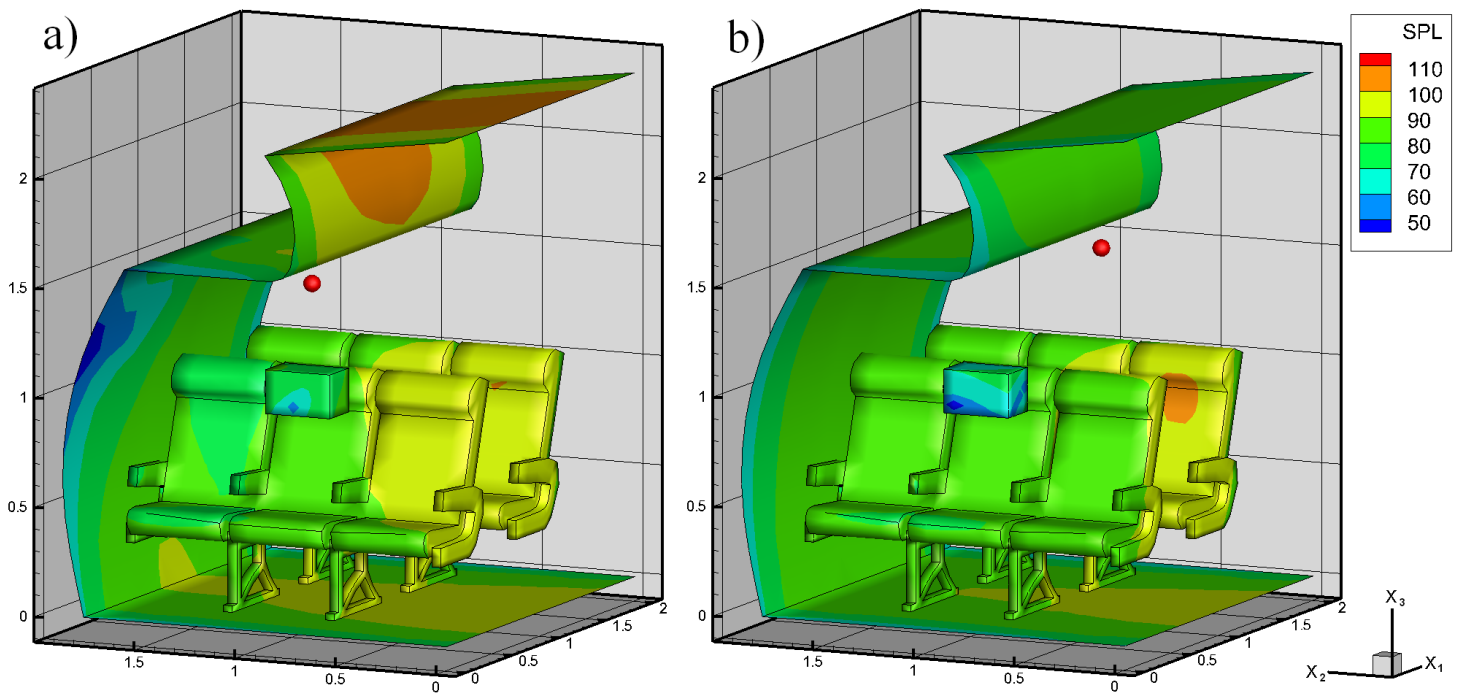

Figure 19. ANC at $104 \mathrm{~Hz}$. SPL generated by the a) initial guess and b) final optimised secondary source location/orientation. (Example 4)

[7] Garcia-Bonito J, Elliott SJ. Active Cancellation of Acoustic Pressure and Particle velocity in the Near Field of a Source. Journal of Sound and Vibration 1999; 221(1), pp. 85-116, DOI: $10.1006 /$ jsvi.1998.1989.

[8] Garcia-Bonito J, Elliott SJ. Local Active Control of Diffracted Diffuse Sound Fields. Journal of the Acoustical Society of America 1995; 98(2), pp. 1017-1024, DOI: 10.1121/1.413666.

[9] Rafaely B, Elliott SJ, Garcia-Bonito J. Broadband performance of an active headrest. Journal of the Acoustical Society of America 1999; 106(2), pp. 787-793, DOI: 10.1121/1.427134.

[10] Padula SL, Palumbo DL, Kincaid RK. Optimal Sensor/Actuator Locations For Active Structural Acoustic Control; 1998; AIAA 98-1865, DOI: 10.1.1.77984.

[11] Christensen ST, Sorokin SV, Olhoff N. On analysis and optimisation in structural acoustics Part i: Problem formulation and solution techniques. - Part ii: Exemplifications for axisymmetric structures. Structural optimisation; 1998; 16, pp. 83-107, DOI: 10.1007/BF01202818, 10.1007/BF01202819.

[12] Marburg S. Developments in structural-acoustic optimisation for passive noise control. Archives of Computational Methods in Engineering. State of the art reviews; 2002; 9(4), pp. 291-370, DOI: 35400060228294.0001. 
[13] Ruckman CE, Fuller CR. Optimizing Actuator Location in Active Noise Control System using Subset Selection. Journal of Sound and Vibration 1995; 172(5), pp. 605-627, DOI: 10.1006/jsvi.1995.0458.

[14] Kincaid RK, Laba K, Padula S. Quelling cabin noise in turboprop aircraft via active control. J. Comb. Optim.; 1997; 1, pp. 229-250, DOI: 10.1023/A:1009724325820.

[15] Baek KH, Elliott SJ. Natural Algorithms For Choosing Source Locations In Active Control Systems. Journal of Sound and Vibration 1995; 186(2), pp. 245-267, DOI: 10.1006/jsvi.1995.0447.

[16] Koopmann G.H., Fahnline J.B. Designing Quiet Structures: A Sound Power minimisation Approach. Academic Press, San Diego, London, 1997.

[17] Martin T, Roure A. Active Noise Control Of Acoustic Sources Using Spherical Harmonics Expansion and a Genetic Algorithm: Simulation and Experiment. Journal of Sound and Vibration 1998; 212 (3), pp. 511-523, DOI: 10.1006/jsvi.1997.1444.

[18] Kincaid RK, Laba K. Reactive Tabu Search and Sensor Selection in Active Structural Acoustic Control Problems. Journal of Heuristics; 1998; 4, pp. 199-220, DOI: 10.1023/A:1009681732632.

[19] Seyedin SA, Abedi MH. Optimizing Secondary Source Location in Acoustic ANC Systems. Information and Communication Technologies ICTTA '06 2006; 1, pp. 1953-1957, DOI: 10.1109/ICTTA.2006.1684689.

[20] Christensen ST, Olhoff N. Shape optimisation of a loudspeaker diaphragm with respect to sound directivity properties. Control and Cybernetics; 1998; 27(2), pp. 177-198.

[21] Brancati A, Aliabadi MH, Benedetti I. Hierarchical Adaptive Cross Approximation GMRES Technique for Solution of Acoustic Problems Using the Boundary Element Method. CMES: Computer Modeling in Engineering 63 Sciences 2009; 43(2), pp. 149-172, DOI: 10.3970/cmes.2009.043.149.

[22] Mellings SC and Aliabadi MH. Flaw identification using the boundary element method. International Journal for Numerical Methods in Engineering 1995; 38(3), pp. 399-419, DOI: $10.1002 /$ nme.1620380304.

[23] Nishimura N and Kobayashi S. A boundary integral equation method for an inverse problem related to crack detection. International Journal for Numerical Methods in Engineering 1995; 32(7), pp. 1371-1387, DOI: 10.1002/nme.1620320702.

[24] Mallardo V, Aliabadi MH. A BEM sensitivity and shape identification analysis for acoustic scattering in fluid-solid problems. International Journal for Numerical Methods in Engineering 1998; 41(8), pp. 1527-1541, DOI: 10.1002/(SICI)1097-0207(19980430)41:8<1527::AIDNME352>3.0.CO;2-O. 
[25] Koo BU, Ih JG, Lee BC. Acoustic shape sensitivity analysis using the boundary integral equation. J. Acoust. Soc. Am. 1998; 104(5), pp. 2851-2860, DOI: 10.1121/1.423869.

[26] Kane JH, Mao S, Everstine GC. A boundary element formulation for acoustic shape sensitivity analysis. Journal of the Acoustical Society 1991; 90(1), pp. 561-573, DOI: 0014966/91/070561-13.

[27] Koo BU. Shape design sensitivity analysis of acoustic problems using a boundary element method. Computers \& Structures 1997; 65(5), pp. 713-719, DOI: 10.1016/S00457949(96)00322-7.

[28] Ciskowski RD, Brebbia CA. Boundary element methods in acoustics. Computational Mechanics Publications Elsevier Applied Science, 1991.

[29] Cunefare KA, Koopmann GH. A boundary element approach to optimisation of active noise control sources on three-dimensional structures. ASME J. Vibr. Acoust. 1990; 113, pp. 387-394, DOI: 10.1115/1.2930196.

[30] Yang TC, Tseng CH. Effective Optimization-Based Approach for Designing Active Noise Control Systems in Enclosures. Finite Elements in Analysis and Design 1994; 15, pp. 303316 .

[31] Bai MR, Chang S. Active Noise Control of Enclosed Harmonic Fields by Using BEM-Based optimisation Techniques. Applied Acoustics 1996; 48(1), pp. 15-32, DOI: 10.1016/0003682X(95)00040-G.

[32] Guru Prasad K, Kane JH. Shape reanalysis and sensitivities utilising preconditioned iterative boundary solvers. Structural optimisation 1992; 4, pp. 224-235, DOI: 10.1007/BF01742749.

[33] Fritze D, Marburg S, Hardtke HJ. FEM-BEM-coupling and structural-acoustic sensitivity analysis for shell geometries. Computers and structures 2005; 83, pp. 143-154, DOI: 10.1016/j.compstruc.2004.05.019.

[34] Nemitz N, Bonnet M. Topological sensitivity and FMM-accelerated BEM applied to 3D acoustic inverse scattering. Engineering Analysis with Boundary Elements 2008; 32(11), pp. 957-970, DOI: 10.1016/j.enganabound.2007.02.006 .

[35] Nelson PA and Elliott SJ. Active Control of' Sound. Academic Press, New York, 1992.

[36] Wrobel LC, Aliabadi MH. The Boundary Element Method. Two volumes set. New Jersey: UK JOHN WILEY, 2002.

[37] Dominguez J. Boundary Elements in Dynamics. Computational Mechanics Publications, 1993.

[38] Nelson PA, Elliott SJ. Active Control of Sound. Press, New York, 1992.

[39] Arora JS. Introduction to optimum design. New York: McGraw-Hill, 1989. 
[40] Press WH. Numerical recipes: the art of scientific computing. Cambridge University Press, 2007.

[41] Crotty JM. A block equation solver for large unsymmetric matrices arising in the boundary integral equation method. International Journal for Numerical Methods in Engineering 1982; 18(7), pp. 997-1017, DOI: 10.1002/nme.1620180705.

[42] Rigby RH, Aliabadi MH. Out-of-core solver for large, multi-zone boundary element matrices. International Journal for Numerical Methods in Engineering 1995; 38(9), pp. 15071533, DOI: 10.1002/nme.1620380905.

[43] Mansur WJ, Araújo FC, Malaghini JEB. Solution of BEM systems of equations via iterative techniques. International Journal for Numerical Methods in Engineering 1992; 33(9), pp. 1823-1841, DOI: 10.1002/nme.1620330905.

[44] Gumerov NA, Duraiswami R. Fast Multipole Methods for the Helmholtz Equation in Three Dimensions. Oxford, UK: Elsevier, 2005.

[45] Bebendorf M, Rjasanow S. Adaptive low-rank approximation of collocation matrices. Computing 2003; 70(1), pp. 1-24, DOI: 10.1007/s00607-002-1469-6.

[46] Benedetti I, Aliabadi MH, Daví G. A fast 3D dual boundary element method based on hierarchical matrices. International Journal of Solids and Structures 2007; 45(7-8), pp. 2355-2376, DOI: 10.1016/j.ijsolstr.2007.11.018.

[47] Bermùdez A, Gamallo P, Rodrìguez R. Finite element methods in local active control of sound. SIAM Journal on Control and optimisation 2004; 43, pp. 437-465.

[48] Brancati A. Boundary Element Method for Fast Solution of Acoustic Problems: Active and Passive Noise Control. PhD Thesis, Imperial College London, 2010.

[49] Lu HY. Effect of Excitation on Coaxial Jet Noise. AIAA Journal 1963; 21(2), pp. 214-220.

[50] David A, Elliott SJ. Numerical Studies of Actively Generated Quiet Zones. Applied Acoustics 1994; 41, pp. 63-79, DOI: 10.1016/0003-682X(94)90085-X.

\section{APPENDIX}

For the sake of completeness the cost function obtained by the proposed procedure and depicted in figure 8 is discussed towards the results obtained by Elliott et al. $[9,50]$. In such papers the trend of a spatial extent of a $10 \mathrm{~dB}$ zone of quiet for a monopole secondary source in a pure diffuse primary field is presented. They show that the extent of the zones of quiet converges to a sphere of diameter $\lambda / 10$ as the distance between the secondary source and the cancellation point is increased. Such a regular trend of the quiet zone extent is not achieved in our first example where an oscillatory trend is obtained, instead (see figure 8). The 
approach in [50] is based upon an idealized model that creates a pure tone diffuse field which is homogeneous in all the directions, whereas in the present contribution the diffuse field is created by a finite number of planewaves. The distribution of the primary waves plays a fundamental role in the optimum CV location.

In figure 20 primary diffuse disturbance created by 72 planewaves is depicted for four different frequencies $(109,273,546,1092 \mathrm{~Hz})$ in a plane of dimension $1 \times 1$ $m^{2}$ meshed with 931 nodes. The same plane has been used in figure 21 .
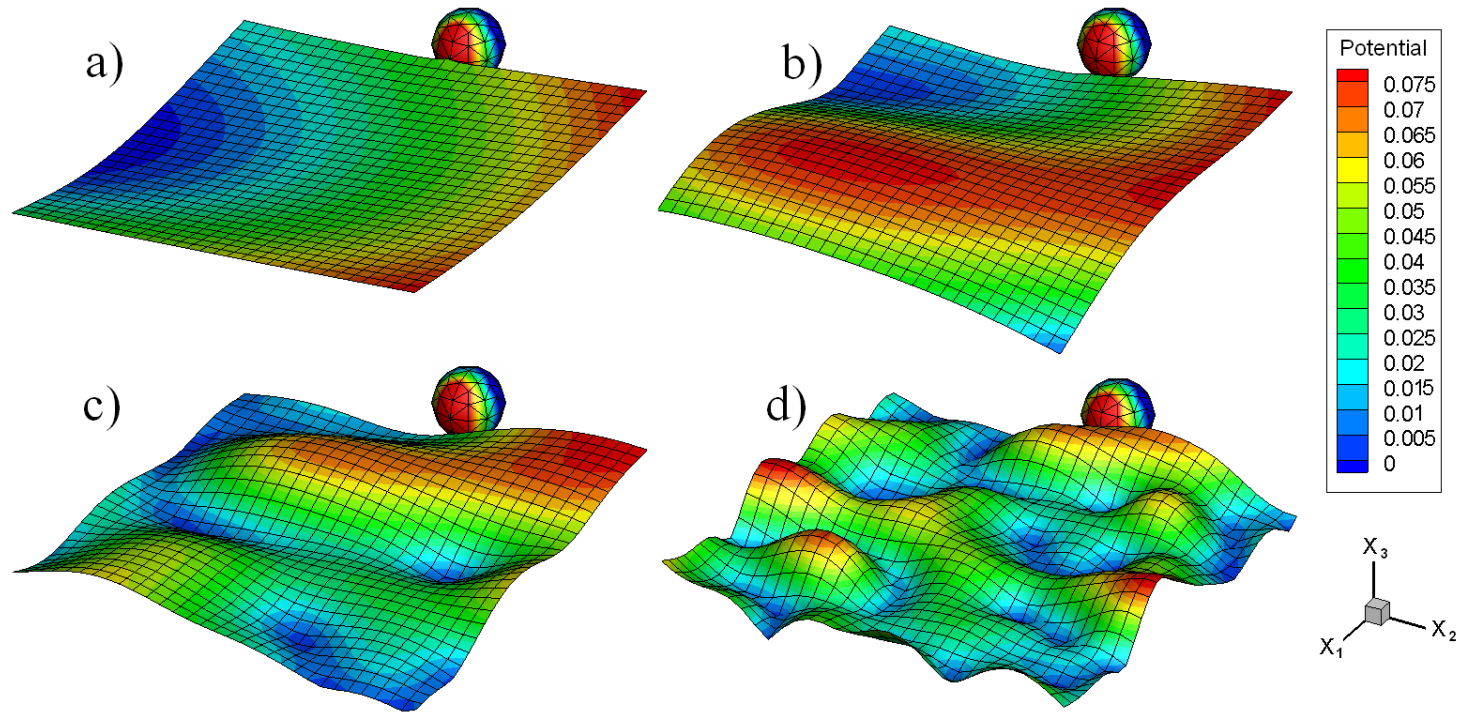

Figure 20. Primary diffuse noise amplitude created by 72 planewaves distributed in all directions of the space for four different frequencies: a) $109 \mathrm{~Hz}$; b) $273 \mathrm{~Hz}$; c) $546 \mathrm{~Hz}$; d) $1092 \mathrm{~Hz}$.

For such a primary diffuse field the cost function trends are shown in figure 8. The troughs and crests represent the optimum and worst locations, respectively, of the $\mathrm{CV}$ along the $x_{1}$-axes for the four frequencies.

Figure 9 shows the total (primary and secondary fields) potential amplitude distribution for one local optimum value (a trough in figure 8) at each frequency, whereas figure 22 shows the same quantity at one of the worst values. 

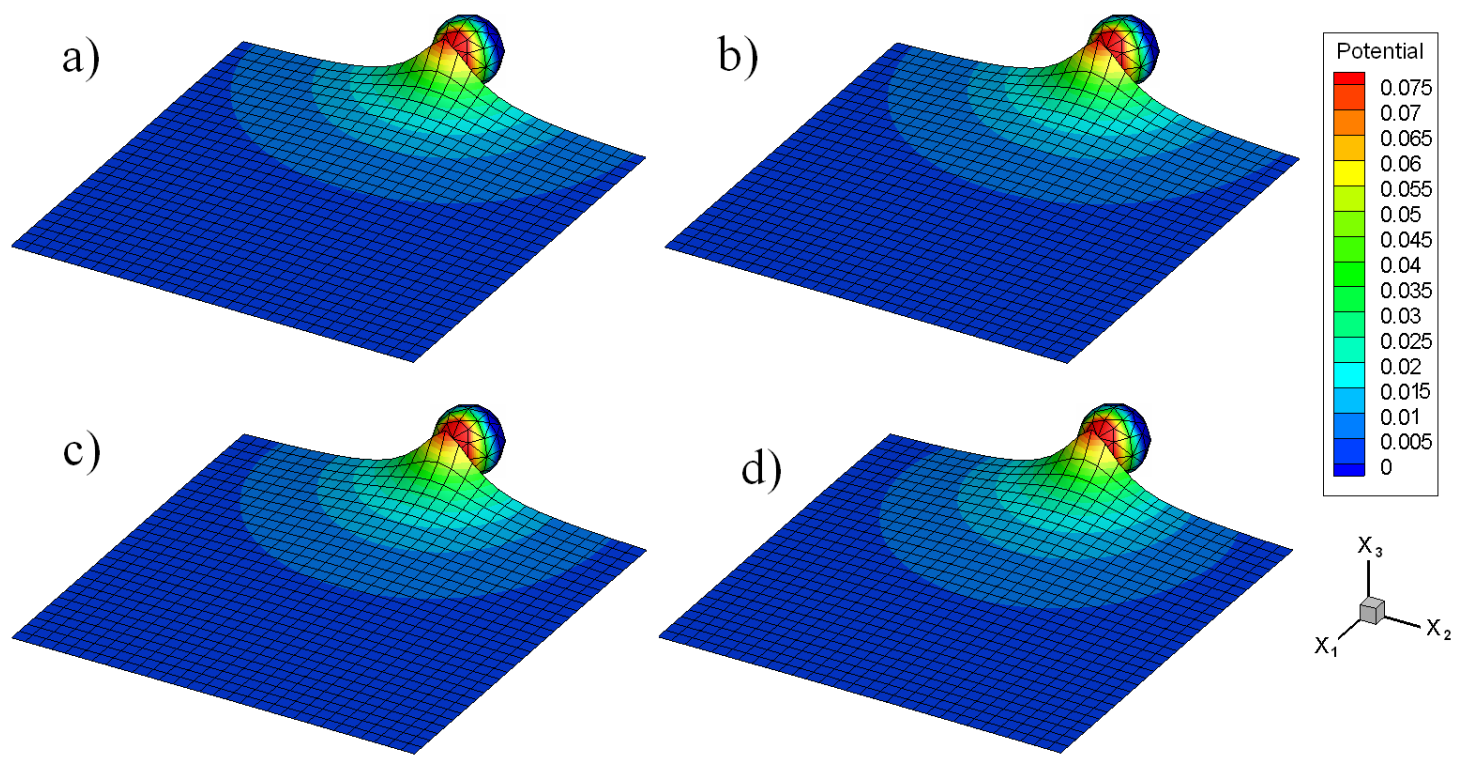

FIGURE 21. Secondary field amplitude created by a rigid sphere with an active segment of $120^{\circ}$ degree and radius $8 \mathrm{~cm}$ for four different frequencies: a) $109 \mathrm{~Hz}$; b) $273 \mathrm{~Hz}$; c) $546 \mathrm{~Hz}$; d) $1092 \mathrm{~Hz}$.

As evident the best CV locations are located where the total fields form a sufficient flat noise reduction distribution, whereas the worst CV locations are placed between two contiguous zones of high noise level. In particular figures 22b) and 22-d) show that a low attenuation CV location can be also placed further away from the secondary source due to the diffuse distribution of the primary noise, highly in contrast with $[9,50]$ results.

It should be pointed out that figures 9, 20, 21 and 22 have been obtained by multiplying the resulting potential for a constant factor, different for each figure, in order to better visualize their variations. 

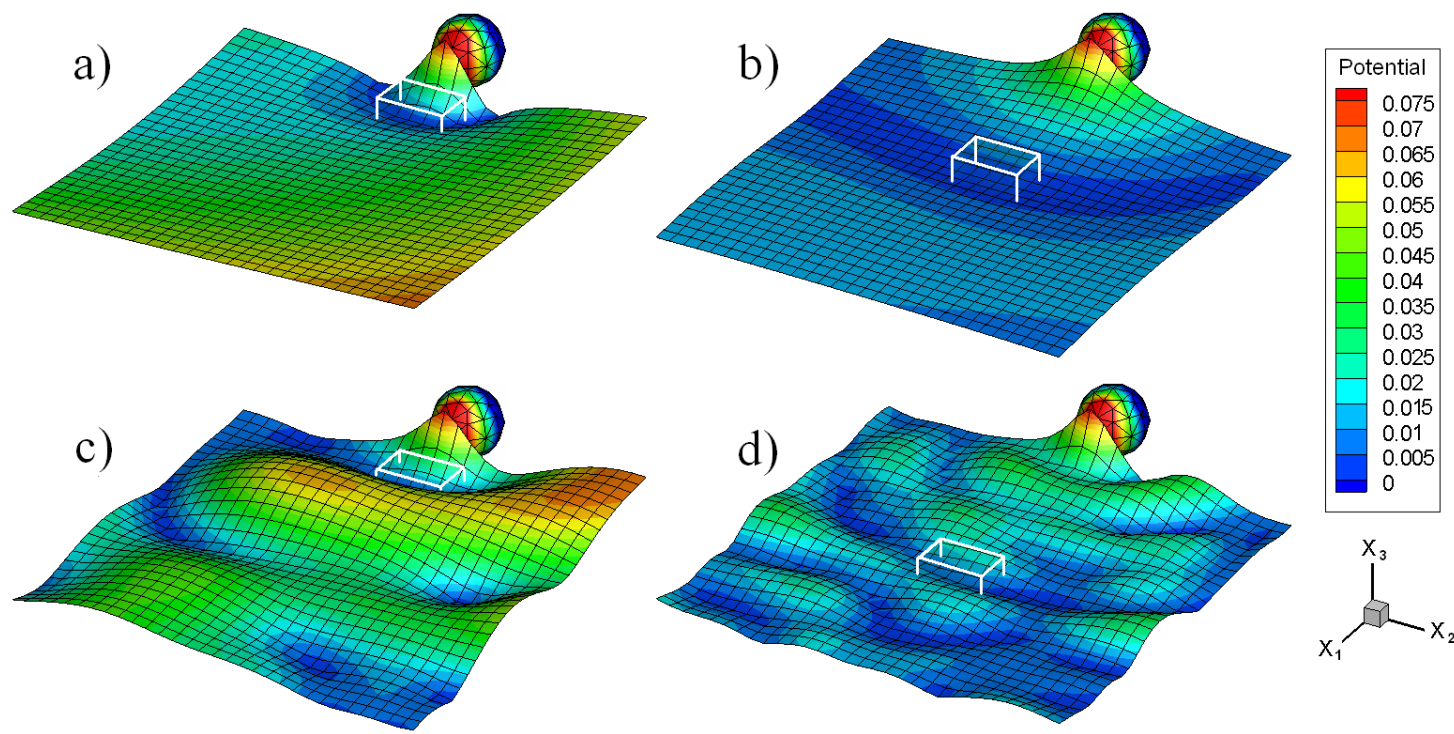

Figure 22. Total amplitude potential of the worst CV $x_{1^{-}}$ locations corresponding to the crests of the cost function in figure 8: a) $109 \mathrm{~Hz}$; b) $273 \mathrm{~Hz}$; c) $546 \mathrm{~Hz}$; d) $1092 \mathrm{~Hz}$. 